\title{
Studies of Inviscid Flux Schemes for Acoustics and Turbulence Problems
}

\author{
C. I. Morris* \\ NASA Marshall Space Flight Center, Huntsville, AL, 35812, USA
}

\begin{abstract}
The last two decades have witnessed tremendous growth in computational power, the development of computational fluid dynamics (CFD) codes which scale well over thousands of processors, and the refinement of unstructured grid-generation tools which facilitate rapid surface and volume gridding of complex geometries. Thus, engineering calculations of $10^{7}-10^{8}$ finite-volume cells have become routine for some types of problems. Although the Reynolds Averaged Navier Stokes (RANS) approach to modeling turbulence is still in extensive and wide use, increasingly large-eddy simulation (LES) and hybrid RANS-LES approaches are being applied to resolve the largest scales of turbulence in many engineering problems. However, it has also become evident that LES places different requirements on the numerical approaches for both the spatial and temporal discretization of the Navier Stokes equations than does RANS. In particular, LES requires high time accuracy and minimal intrinsic numerical dispersion and dissipation over a wide spectral range. In this paper, the performance of both central-difference and upwind-biased spatial discretizations is examined for a one-dimensional acoustic standing wave problem, the Taylor-Green vortex problem, and the turbulent channel flow problem.
\end{abstract}

\section{Nomenclature}

a sound speed

$E \quad$ specific total energy, $e+E_{k}$

$E_{k} \quad$ kinetic energy, $U^{2} / 2$

$e \quad$ specific internal energy

f body force per unit volume vector, $\left[f_{x}, f_{y}, f_{z}\right]^{T}$

$\mathbf{F}, \mathbf{G}, \mathbf{H} \quad$ inviscid flux vectors in the $x-, y$-, and $z$-direction, respectively

$\mathbf{F}_{\mathbf{v}}, \mathbf{G}_{\mathbf{v}}, \mathbf{H}_{\mathbf{v}}$ viscous flux vectors in the $x-, y$-, and $z$-direction, respectively

$H \quad$ specific total enthalpy, $e+E_{k}+p / \rho$

$h \quad$ channel half-height, reference length for channel flow problem

$k \quad$ scaled wavenumber, $2 \pi n / N$

$l \quad$ reference length for one-dimensional acoustic and Taylor-Green vortex problems

$M \quad$ Mach number, $U / a$

$\dot{m} \quad$ mass flow rate

$m \quad$ subiteration level in Runge-Kutta time-advancement

$N \quad$ number of grid points in a particular direction of a domain

$n$ number of wavelengths in a particular direction of a domain, also time-advancement level

$p \quad$ pressure

$Q \quad$ Q-criterion, $(\mathbf{R}: \mathbf{R}-\mathbf{S}: \mathbf{S}) / 2$

$\mathbf{q} \quad$ heat flux vector, $\left[q_{x}, q_{y}, q_{z}\right]^{T}$

$R \quad$ gas constant

$\mathbf{R} \quad$ rotation-rate tensor, $\left(\nabla \mathbf{u}-\nabla \mathbf{u}^{T}\right) / 2$

S strain-rate tensor, $\left(\nabla \mathbf{u}+\nabla \mathbf{u}^{T}\right) / 2$

$T \quad$ temperature

${ }^{*}$ Lead Aerospace Engineer, Aerosciences Branch/EV33, Senior Member AIAA. 


$\begin{array}{ll}t & \text { time } \\ \mathbf{U} & \text { conservative variable state vector } \\ U & \text { velocity magnitude, }\|\mathbf{u}\| \\ \mathbf{u} & \text { velocity vector, }[u, v, w]^{T} \\ V & \text { volume } \\ \mathbf{W} & \text { source vector accounting for body forces } \\ u, v, w & \text { components of velocity vector in the } x \text {-, } y \text {-, and } z \text {-direction, respectively } \\ x, y, z & \text { cartesian coordinates }\end{array}$

\section{Subscripts}

$\tau$

denotes an initial condition

denotes a wall friction property

denotes a flow property at the centerline

computational indices in the $x-, y$-, and $z$-direction, respectively

denotes a mean flow property

denotes a flow property at the wall

Symbols

$\lambda$

$\mu$

$\omega$

$\Omega$

$\phi$

$\rho$

$\tau$

$\varepsilon$

ratio of specific heats

thermal conductivity

viscosity

vorticity vector, $\left[\omega_{x}, \omega_{y}, \omega_{z}\right]^{T}$

enstrophy, $\boldsymbol{\omega} \cdot \boldsymbol{\omega} / 2$

generic flowfield quantity

density

viscous stress tensor

turbulent kinetic energy dissipation rate

Superscripts

* $\quad$ variable normalized by a characteristic flow timescale

$+\quad$ variable normalized by wall friction variables

\section{Introduction}

$\mathrm{T}$ URBULENCE is a critical factor in most aero- and propulsion-related flows. For many years, the industrystandard approach to incorporating the effect of turbulence in computational fluid dynamics (CFD) calculations has been the Reynolds Averaged Navier Stokes (RANS) modeling approach. RANS implicitly time-averages turbulent motion, and models its effect on the mixing of species, momentum and energy in a flow field. The approach is economical and often allows a CFD calculation to proceed to steadystate. However, it makes several simplifying assumptions, and the results will only be as good as the RANS turbulence model. Meanwhile, the last two decades have witnessed rapid and explosive growth in computational power, the development of CFD codes which scale well over thousands of processors, and the refinement of unstructured grid-generation tools which facilitate rapid surface and volume gridding of complex geometries. CFD engineering calculations of $10^{7}-10^{8}$ finite-volume cells have become routine. Thus, large eddy simulation (LES), which attempts to directly resolve the unsteady motion of the largest scales of turbulence, is increasingly of interest for many fluids engineering problems. ${ }^{1-3}$ Additionally, hybrid RANS-LES approaches such as Spalart's Detached Eddy Simulation (DES) ${ }^{4}$ which attempt to marry the best strengths of both methods, are in active use and development.

Because it seeks to accurately resolve the unsteady motion of the largest scales of turbulence, LES places certain requirements on the numerical method used to discretize and advance the Navier Stokes equations. In particular, LES requires high time accuracy and minimal intrinsic numerical dispersion and dissipation over a wide range of length scales. With respect to the spatial discretization, central difference methods, particularly higher-order ones, can satisfy this requirement. However, the stability of these methods on realistic engineering meshes, particular unstructured meshes, can be very problematic. Upwind schemes, on the other hand, are typically very robust on engineering meshes and enjoy wide use in many production CFD codes. These schemes have been very successful in RANS calculations, but their suitability for LES has 
been arguable for several years. Mittal and Moin ${ }^{5}$ compared a $2^{\text {nd }}$ order central difference and a $5^{\text {th }}$ order upwind scheme on LES of flow behind a circular cylinder at a Reynolds number of 3900, and concluded that the upwind scheme significantly dissipated the small scales in the wake. Garnier et al. ${ }^{6}$ studied the ability of several different upwind shock-capturing schemes to properly resolve near-incompressible and compressible decaying isotropic turbulence, and found that while the higher-order shock-capturing schemes did reproduce some aspects of turbulence, they were still overly dissipative for this problem. Mossi and Sagaut ${ }^{7}$ studied the characteristics of several different schemes in DNS and LES of subsonic and supersonic turbulent channel flow at a wall-variable Reynolds number of 180. A $2^{\text {nd }}$ order central difference method did best out of the schemes studied, while a $3^{\text {th }}$ order upwind scheme was again overly dissipative. Park et al. ${ }^{8}$ compared high-order central and upwind schemes on several different flow problems and reached similar conclusions. A common conclusion from these studies is that LES sub-grid scale (SGS) turbulence models, while needed for LES using higher-order central difference schemes, are unnecessary for simulations using upwind schemes, and in fact typically reduce accuracy at increased cost.

Although much research in DNS and LES has taken place assuming incompressible fluid flow, the interest in this work is in spatial discretizations intended for compressible flow due to the wider range of applicability for aerospace flowfields. Ducros et al. ${ }^{9}$ developed stable second, fourth and sixth-order skew-symmetric-like central difference schemes for compressible flows. The same author, in Ref.10, developed a flowfield sensor suitable for discriminating shocks amid compressible turbulence. Honein and Moin ${ }^{11}$ examined the skewsymmetric central differencing approach for several different treatments for the conservation of energy and entropy. Recently, a large number of additional studies in the literature have investigated the suitability of various central difference, upwind-based, and hybrid schemes for LES of compressible turbulence in the presence of shock waves. Due to space considerations, only a few will be mentioned here. Larson et al. ${ }^{12}$ found that a hybrid approach using skew-symmetric central difference schemes for the bulk of the flowfield, and upwind schemes near shock waves, can be well worth the complexity introduced. Similar conclusions were reached in a comprehensive study by Johnsen et al. ${ }^{13}$ The recent review by Pirozzoli ${ }^{14}$ summarizes much of the work to date.

The work reported in this paper grew out of the author's work on modeling supersonic film cooling using RANS. ${ }^{15}$ Subsequent attempts to model the test cases examined in that work using a hybrid RANS-LES approach with an unstructured upwind-based production CFD code were problematic, and this study was initiated by the author's attempt to assimilate and understand the above references. The purpose of this paper is to evaluate the suitability of several different central difference and upwind inviscid flux schemes for a simple one-dimensional acoustic standing wave problem, as well as DNS and LES of the Taylor-Green vortex problem and a turbulent channel flow problem. The emphasis in this particular work is on the characteristics of the inviscid flux schemes themselves, rather than the coupling of various schemes with SGS turbulence models. As such, although the grid resolution in the two turbulence problems ranges from DNS levels to fine-grid LES levels, no explicit SGS models are included in the calculations. Additionally, although the compressible form of the Navier-Stokes equations are solved, all of test cases occur at low subsonic flow speeds, suitable for comparison with well-established results from analysis or the literature.

\section{Governing Equations}

The compressible Navier-Stokes equations for a single chemical species are the governing model for this study. The time-dependent differential form of these equations in cartesian coordinates is written as follows:

$$
\frac{\partial \mathbf{U}}{\partial t}+\frac{\partial\left(\mathbf{F}-\mathbf{F}_{\mathbf{v}}\right)}{\partial x}+\frac{\partial\left(\mathbf{G}-\mathbf{G}_{\mathbf{v}}\right)}{\partial y}+\frac{\partial\left(\mathbf{H}-\mathbf{H}_{\mathbf{v}}\right)}{\partial z}=\mathbf{W}
$$

where the state vector, $\mathbf{U}$, and the source vector accounting for body forces, $\mathbf{W}$ are given by

$$
\mathbf{U}=\left[\begin{array}{c}
\rho \\
\rho u \\
\rho v \\
\rho w \\
\rho E
\end{array}\right], \quad \mathbf{W}=\left[\begin{array}{c}
0 \\
f_{x} \\
f_{y} \\
f_{z} \\
f_{x} u+f_{y} v+f_{z} w
\end{array}\right]
$$


and the inviscid flux vectors, $\mathbf{F}, \mathbf{G}$ and $\mathbf{H}$, and the viscous flux vectors $\mathbf{F}_{\mathbf{v}}, \mathbf{G}_{\mathbf{v}}$ and $\mathbf{H}_{\mathbf{v}}$ are given by

$$
\begin{aligned}
& \mathbf{F}=\left[\begin{array}{c}
\rho u \\
\rho u u+p \\
\rho u v \\
\rho u w \\
\rho u H
\end{array}\right], \quad \mathbf{F}_{\mathbf{v}}=\left[\begin{array}{c}
0 \\
\tau_{x x} \\
\tau_{x y} \\
\tau_{x z} \\
u \tau_{x x}+v \tau_{x y}+w \tau_{x z}-q_{x}
\end{array}\right], \\
& \mathbf{G}=\left[\begin{array}{c}
\rho v \\
0 \\
\rho v v+p \\
\rho v w \\
\rho v H
\end{array}\right], \quad \mathbf{G}_{\mathbf{v} x}=\left[\begin{array}{c}
\tau_{y y} \\
\tau_{y z} \\
\rho w \\
\rho w u \\
\rho w v \\
\rho w w+p \\
\rho w H
\end{array}\right], \quad \mathbf{H}_{\mathbf{v}}=\left[\begin{array}{c}
u \tau_{y x}+w \tau_{y z}-q_{y} \\
0 \\
\tau_{z x} \\
\tau_{z y} \\
\tau_{z z}
\end{array}\right], \\
& \mathbf{H}=\left[\begin{array}{c}
u \tau_{y y}+w \tau_{z z}-q_{z}
\end{array}\right],
\end{aligned}
$$

where the components of the viscous shear stress tensor, $\boldsymbol{\tau}$, are given by

$$
\begin{gathered}
\tau_{x x}=\frac{2}{3} \mu\left(2 \frac{\partial u}{\partial x}-\frac{\partial v}{\partial y}-\frac{\partial w}{\partial z}\right), \quad \tau_{y y}=\frac{2}{3} \mu\left(2 \frac{\partial v}{\partial y}-\frac{\partial u}{\partial x}-\frac{\partial w}{\partial z}\right), \quad \tau_{z z}=\frac{2}{3} \mu\left(2 \frac{\partial w}{\partial z}-\frac{\partial u}{\partial x}-\frac{\partial v}{\partial y}\right), \\
\tau_{x y}=\mu\left(\frac{\partial u}{\partial y}+\frac{\partial v}{\partial x}\right)=\tau_{y x}, \quad \tau_{x z}=\mu\left(\frac{\partial w}{\partial x}+\frac{\partial u}{\partial z}\right)=\tau_{z x}, \quad \tau_{y z}=\mu\left(\frac{\partial v}{\partial z}+\frac{\partial w}{\partial y}\right)=\tau_{z y}
\end{gathered}
$$

and the heat flux vector as

$$
q_{x}=-\lambda \frac{\partial T}{\partial x}, \quad q_{y}=-\lambda \frac{\partial T}{\partial y}, \quad q_{z}=-\lambda \frac{\partial T}{\partial z}
$$

A calorically perfect ideal gas equation of state is used: $p=\rho R T$, and $T=(\gamma-1) e / R \cdot \gamma=1.4$ and $R=287 \mathrm{~J} / \mathrm{Kg}-\mathrm{K}$ for all cases studied in this work. The transport properties $\mu$ and $\lambda$ are calculated using the Sutherland formulas for air ${ }^{16}$

$$
\begin{gathered}
\mu=1.716 \times 10^{-5} \mathrm{~Pa}-\mathrm{s}\left(\frac{T}{273 \mathrm{~K}}\right)^{3 / 2} \frac{273 \mathrm{~K}+111 \mathrm{~K}}{T+111 \mathrm{~K}} \\
\lambda=2.41 \times 10^{-2} \mathrm{~W} / \mathrm{m}-\mathrm{K}\left(\frac{T}{273 \mathrm{~K}}\right)^{3 / 2} \frac{273 \mathrm{~K}+194 \mathrm{~K}}{T+194 \mathrm{~K}}
\end{gathered}
$$

\section{Numerical Method}

The Navier-Stokes equations are solved by the finite-difference method in the conventional cartesian coordinate system. The inviscid flux terms $\mathbf{F}, \mathbf{G}$ and $\mathbf{H}$ are the portion of the equations that deal with the convection of mass, momentum and energy. Historically, the discretization of these terms has been a driving issue in CFD research since its inception. Four central difference (denoted as CD-2, CD-4, CD-6 and CD-8) schemes and four upwind-biased (denoted as UB-1, UB-3, UB-5, UB-7) schemes are evaluated in this work. The number in the name for each scheme denotes the formal order of accuracy. As will be shown below, the dispersion (phase) error properties of each central difference scheme is the same as the upwind-biased scheme of one less order of accuracy (e.g., CD-4 has the same dispersion error as UB-3). Additionally, an upwind scheme based on the Fromm scheme (denoted UF-2) is included, as well as a corresponding central difference scheme (CF-2) with the same dispersion error properties. UF-2 should be similar to the linear slope reconstructions used in many practical unstructured CFD codes. Finally, a compact-upwind scheme 
(denoted CU-5) from the work of Pirozzoli ${ }^{17}$ is included. Conservative differencing was used such that, at node $i, j, k$, the flux derivatives in the $x$-direction is given by

$$
\frac{\partial \mathbf{F}_{i, j, k}}{\partial x}=\frac{\mathbf{F}_{i+\frac{1}{2}, j, k}-\mathbf{F}_{i-\frac{1}{2}, j, k}}{\Delta x}
$$

where $\mathbf{F}_{i+\frac{1}{2}, j, k}$ and $\mathbf{F}_{i-\frac{1}{2}, j, k}$ are interpolated and formed from the surrounding flowfield points. The same process is used for the flux derivatives of $\mathbf{G}$ and $\mathbf{H}$ in the $y$ - and $z$-directions, respectively.

We now turn to specifying how the flux values at the half-nodes are formed for central differencing. Note that the inviscid flux terms $\mathbf{F}, \mathbf{G}$ and $\mathbf{H}$ contain combinations of several different primitive flow variables. In divergence form central differencing, the flux values themselves are directly differenced. In skew-symmetric form central differencing, the flux is formed from a combination of central differences of the flux values and the component flow property variables themselves. In addition to the work in Refs. 9 and 11, this approach has been investigated for compressible flow by several researchers in recent years. Kennedy and Gruber $^{18}$ developed a skew-symmetric central difference scheme intended to be suitable for strong density variations. Subareddy and Candler ${ }^{19}$ developed a skew-symmetric scheme which maintained consistency between the kinetic energy in the momentum and energy fluxes. An especially attractive feature of all these schemes is that they preserve kinetic energy, resulting in much greater robustness than divergence form central differencing. Pirozzoli in Refs. 20 and 21 showed how several different skew-symmetric schemes could be recast in a conservative differencing form. Assuming constant $j$ and $k$ (not shown), and discretization in the $i$-direction, his methodology yields

$$
\begin{array}{rlrl}
\text { CD-2: } & \mathbf{F}_{i+\frac{1}{2}} & =\mathbf{F}_{\mathrm{avg}}(i, i+1) \\
\mathrm{CD}-4: & & \mathbf{F}_{i+\frac{1}{2}} & =\frac{4}{3} \mathbf{F}_{\mathrm{avg}}(i, i+1)-\frac{1}{6}\left[\mathbf{F}_{\mathrm{avg}}(i-1, i+1)+\mathbf{F}_{\mathrm{avg}}(i, i+2)\right] \\
& \mathbf{F}_{i+\frac{1}{2}} & =\frac{3}{2} \mathbf{F}_{\mathrm{avg}}(i, i+1)-\frac{3}{10}\left[\mathbf{F}_{\mathrm{avg}}(i-1, i+1)+\mathbf{F}_{\mathrm{avg}}(i, i+2)\right] \\
& & & \frac{1}{30}\left[\mathbf{F}_{\mathrm{avg}}(i-2, i+1)+\mathbf{F}_{\mathrm{avg}}(i-1, i+2)+\mathbf{F}_{\mathrm{avg}}(i, i+3)\right] \\
\mathrm{CD}-6: & \mathbf{F}_{i+\frac{1}{2}} & =\frac{16}{10} \mathbf{F}_{\mathrm{avg}}(i, i+1)-\frac{4}{10}\left[\mathbf{F}_{\mathrm{avg}}(i-1, i+1)+\mathbf{F}_{\mathrm{avg}}(i, i+2)\right] \\
& +\frac{8}{105}\left[\mathbf{F}_{\mathrm{avg}}(i-2, i+1)+\mathbf{F}_{\mathrm{avg}}(i-1, i+2)+\mathbf{F}_{\mathrm{avg}}(i, i+3)\right] \\
\mathrm{CD}-8: & & & \frac{1}{140}\left[\mathbf{F}_{\mathrm{avg}}(i-3, i+1)+\mathbf{F}_{\mathrm{avg}}(i-2, i+2)+\mathbf{F}_{\mathrm{avg}}(i-1, i+3)+\mathbf{F}_{\mathrm{avg}}(i, i+4)\right] \\
\mathrm{CF}-2: & \mathbf{F}_{i+\frac{1}{2}} & =\frac{3}{2} \mathbf{F}_{\mathrm{avg}}(i, i+1)-\frac{1}{4}\left[\mathbf{F}_{\mathrm{avg}}(i-1, i+1)+\mathbf{F}_{\mathrm{avg}}(i, i+2)\right]
\end{array}
$$

where $\mathbf{F}_{\text {avg }}(i 1, i 2)$ is formed from a symmetric average of all of the individual components of $\mathbf{F}$. As shown in Ref. 20, the form of $\mathbf{F}_{\mathrm{avg}}(i 1, i 2)$ which reproduces the skew-symmetric scheme of Kennedy and Gruber ${ }^{18}$ is

$$
\mathbf{F}_{\text {avg }}(i 1, i 2)=\frac{1}{2}\left(\rho_{i 1}+\rho_{i 2}\right) \frac{1}{2}\left(u_{i 1}+u_{i 2}\right)\left[\begin{array}{c}
1 \\
\frac{1}{2}\left(u_{i 1}+u_{i 2}\right) \\
\frac{1}{2}\left(v_{i 1}+v_{i 2}\right) \\
\frac{1}{2}\left(w_{i 1}+w_{i 2}\right) \\
\frac{1}{2}\left(H_{i 1}+H_{i 2}\right)
\end{array}\right]+\left[\begin{array}{c}
\frac{1}{2}\left(p_{i 1}+p_{i 2}\right) \\
\end{array}\right]
$$

It is interesting to note that if $\mathbf{F}_{\text {avg }}(i 1, i 2)=\frac{1}{2}\left(\mathbf{F}_{i 1}+\mathbf{F}_{i 2}\right)$ then the classic divergence form central difference formulas result, though they can be simplified considerably from the form given in Eqs. (9)-(13). The divergence form was tested in the course of this study to confirm the well-known instability characteristics of the method. Additionally, other skew-symmetric schemes were tested satisfactorily, including a form equivalent to that of Ref. 19. However, unless otherwise noted, all central difference results reported in this paper use the Kennedy and Gruber flux in Eq. (14). The flux values for $\mathbf{G}_{i, j+\frac{1}{2}, k}$ and $\mathbf{H}_{i, j, k+\frac{1}{2}}$, formed at the half-nodes in the $j$ - and $k$-direction respectively, are formed in the same way.

Upwinding can be implemented by various methods in a CFD code. In addition to the work in Ref.17, high order upwind schemes for compressible flow have been developed by Adams and Shariff, ${ }^{22}$ Zhong, ${ }^{23}$ 
Gerolymos et al., ${ }^{24}$ and Rehman et al., ${ }^{25}$ to name only a few. In this work, first a leftward-biased interpolation for the primitive flow variables $\phi=\rho, u, v, w$, and $p$ at the half-node is formed. Again assuming constant $j$ and $k$ (not shown), and discretization in $i$-direction, then

UB-1: $\quad \phi_{i+\frac{1}{2}, L}=\phi_{i}$

UB-3: $\quad \phi_{i+\frac{1}{2}, L}=\left(-\phi_{i-1}+5 \phi_{i}+2 \phi_{i+1}\right) / 6$

UB-5: $\quad \phi_{i+\frac{1}{2}, L}=\left(2 \phi_{i-2}-13 \phi_{i-1}+47 \phi_{i}+27 \phi_{i+1}-3 \phi_{i+2}\right) / 60$

UB-7: $\quad \phi_{i+\frac{1}{2}, L}=\left(-6 \phi_{i-3}+50 \phi_{i-2}-202 \phi_{i-1}+638 \phi_{i}+428 \phi_{i+1}-76 \phi_{i+2}+8 \phi_{i+3}\right) / 840$

$\mathrm{UF}-2: \quad \phi_{i+\frac{1}{2}, L}=\left(-\phi_{i-1}+4 \phi_{i}+\phi_{i+1}\right) / 4$

CU-5: $3 \phi_{i-\frac{1}{2}, L}+6 \phi_{i+\frac{1}{2}, L}+\phi_{i+\frac{3}{2}, L}=\left(\phi_{i-1}+19 \phi_{i}+10 \phi_{i+1}\right) / 3$

The remaining state variables are calculated using the perfect gas law, and the corresponding conservative variables $\mathbf{U}_{i+\frac{1}{2}}$ and the inviscid flux term $\mathbf{F}_{i+\frac{1}{2}}$ are then calculated from these components. The process is then repeated for a rightward-biased interpolation for the half-node using a reflected stencil. For example, $\phi_{i+\frac{1}{2}, R}=\left(2 \phi_{i}+5 \phi_{i+1}-\phi_{i+2}\right) / 6$ for UB-3, and $\phi_{i-\frac{1}{2}, R}+6 \phi_{i+\frac{1}{2}, R}+3 \phi_{i+\frac{3}{2}, R}=\left(10 \phi_{i}+19 \phi_{i+1}+\phi_{i+2}\right) / 3$ for CU-5. The final value of the inviscid flux at the half-node is provided by the Roe approximate Riemann solver, ${ }^{26}$ using these two separate left and right states.

$$
\mathbf{F}_{i+1 / 2}=\frac{1}{2}\left[\mathbf{F}_{i+\frac{1}{2}, L}+\mathbf{F}_{i+\frac{1}{2}, R}\right]-\frac{1}{2}\left|\mathbf{A}_{\text {roe }}\right|\left(\mathbf{U}_{i+\frac{1}{2}, R}-\mathbf{U}_{i+\frac{1}{2}, L}\right)
$$

where $\mathbf{A}_{\text {roe }}$ is the Roe-averaged flux Jacobian matrix.

The viscous terms are discretized using $2^{\text {nd }}$ order central differences. For example, in forming $\mathbf{F}_{\mathbf{v} i+\frac{1}{2}, j, k}$, we need to calculate $\tau_{x x}, \tau_{x y}, \tau_{x z}$ and $q_{x}$ at the half-node $i+\frac{1}{2}, j, k$. The $x$-derivative $\partial u / \partial x$ at $i+\frac{1}{2}, j, k$ is calculated from $\left(u_{i+1}-u_{i}\right) / \Delta x$, and similarly for $\partial v / \partial x, \partial w / \partial x$ and $\partial T / \partial x$. The cross derivative $\partial u / \partial y$ at $i+\frac{1}{2}, j, k$ is found by averaging the derivative computed at the $i$ and $i+1$ nodes, $\partial u / \partial y=\left(u_{i, j+1, k}-\right.$ $\left.u_{i, j-1, k}+u_{i+1, j+1, k}-u_{i+1, j-1, k}\right) / 4 \Delta y$, and similarly for $\partial u / \partial z, \partial v / \partial y$ and $\partial w / \partial z$. The transport coefficients at $i+\frac{1}{2}, j, k$ are also obtained by a simple average of the values at the $i$ and $i+1$ nodes. The viscous fluxes $\mathbf{G}_{\mathbf{v} i, j+\frac{1}{2}, k}$ and $\mathbf{H}_{\mathbf{v} i, j, k+\frac{1}{2}}$, formed at the half-nodes in the $j$ - and $k$-direction respectively, are calculated in an analogous fashion.

All schemes were advanced using a low-storage 4 -stage, $2^{\text {nd }}$ order accurate Runge-Kutta time advancement algorithm

$$
\begin{aligned}
\mathbf{U}_{i, j, k}^{(0)} & =\mathbf{U}_{i, j, k}^{n} \\
\mathbf{U}_{i, j, k}^{(m)} & =\mathbf{U}_{i, j, k}^{(0)}-\frac{1}{5-m} \Delta t \mathbf{R}_{i, j, k}^{(m-1)}, \quad m=1, \ldots, 4 \\
\mathbf{U}_{i, j, k}^{n+1} & =\mathbf{U}_{i, j, k}^{(4)}
\end{aligned}
$$

where

$$
\begin{aligned}
\mathbf{R}_{i, j, k}^{(m-1)} & =\frac{\left(\mathbf{F}-\mathbf{F}_{\mathbf{v}}\right)_{i+\frac{1}{2}, j, k}^{(m-1)}-\left(\mathbf{F}-\mathbf{F}_{\mathbf{v}}\right)_{i-\frac{1}{2}, j, k}^{(m-1)}}{\Delta x}+\frac{\left(\mathbf{G}-\mathbf{G}_{\mathbf{v}}\right)_{i, j+\frac{1}{2}, k}^{(m-1)}-\left(\mathbf{G}-\mathbf{G}_{\mathbf{v}}\right)_{i, j-\frac{1}{2}, k}^{(m-1)}}{\Delta y} \\
& +\frac{\left(\mathbf{H}-\mathbf{H}_{\mathbf{v}}\right)_{i, j, k+\frac{1}{2}}^{(m-1)}-\left(\mathbf{H}-\mathbf{H}_{\mathbf{v}}\right)_{i, j, k-\frac{1}{2}}^{(m-1)}}{\Delta z}-\mathbf{S}_{i, j, k}^{(m-1)}
\end{aligned}
$$

\section{Fourier Analysis of Schemes}

Before proceeding to the numerical results, it is instructive to review the fourier properties of the various numerical schemes for a simple scalar variable $\phi$ discretized into $N$ points over a periodic one-dimensional domain, $0 \leq x \leq l$. Following Ref. 27, we assume a sinusoidal trial solution $\phi(x)=\exp (\iota k x / \Delta x)$, where $\iota=\sqrt{-1}$ and $\Delta x=l / N$. For $n$ wavelengths in the domain, the scaled wavenumber $k$ is equal to $2 \pi n / N$, such that it is equal to $\pi$ at the spatial Nyquist frequency. The exact solution to the first derivative is $\partial \phi / \partial x=(\iota k / \Delta x) \exp (\iota k x / \Delta x)$. For a single variable, $\phi$, if the central difference schemes (Eqs. (9)-(13)) are 
substituted into Eq. (8), the resulting first derivative of $\phi$ becomes

$$
\begin{aligned}
\text { CD-2: } \quad \frac{\partial \phi_{i}}{\partial x}=\frac{1}{2 \Delta x}\left(-\phi_{i-1}+\phi_{i+1}\right) \\
\text { CD-4: } \quad \frac{\partial \phi_{i}}{\partial x}=\frac{1}{12 \Delta x}\left(\phi_{i-2}-8 \phi_{i-1}+8 \phi_{i+1}-\phi_{i+2}\right) \\
\text { CD-6: } \quad \frac{\partial \phi_{i}}{\partial x}=\frac{1}{60 \Delta x}\left(-\phi_{i-3}+9 \phi_{i-2}-45 \phi_{i-1}+45 \phi_{i+1}-9 \phi_{i+2}+\phi_{i+3}\right) \\
\text { CD-8: } \quad \frac{\partial \phi_{i}}{\partial x}=\frac{1}{840 \Delta x}\left(3 \phi_{i-4}-32 \phi_{i-3}+168 \phi_{i-2}-672 \phi_{i-1}+672 \phi_{i+1}-168 \phi_{i+2}+32 \phi_{i+3}-3 \phi_{i+4}\right) \\
\text { CF-2: } \quad \frac{\partial \phi_{i}}{\partial x}=\frac{1}{8 \Delta x}\left(\phi_{i-2}-6 \phi_{i-1}+6 \phi_{i+1}-\phi_{i+2}\right)
\end{aligned}
$$

Similarly, the upwind schemes (Eqs. (15)-(20)) substituted into Eq. (8) result in

$\mathrm{UB}-1: \quad \frac{\partial \phi_{i}}{\partial x}=\frac{1}{\Delta x}\left(-\phi_{i-1}+\phi_{i}\right)$

UB-3: $\quad \frac{\partial \phi_{i}}{\partial x}=\frac{1}{6 \Delta x}\left(\phi_{i-2}-6 \phi_{i-1}+3 \phi_{i}+2 \phi_{i+1}\right)$

$\mathrm{UB}-5: \quad \frac{\partial \phi_{i}}{\partial x}=\frac{1}{60 \Delta x}\left(-2 \phi_{i-3}+15 \phi_{i-2}-60 \phi_{i-1}+20 \phi_{i}+30 \phi_{i+1}-3 \phi_{i+2}\right)$

UB-7: $\quad \frac{\partial \phi_{i}}{\partial x}=\frac{1}{840 \Delta x}\left(6 \phi_{i-4}-56 \phi_{i-3}+252 \phi_{i-2}-840 \phi_{i-1}+210 \phi_{i}+504 \phi_{i+1}-84 \phi_{i+2}+8 \phi_{i+3}\right)$

$\mathrm{UF}-2: \quad \frac{\partial \phi_{i}}{\partial x}=\frac{1}{4 \Delta x}\left(\phi_{i-2}-5 \phi_{i-1}+3 \phi_{i}+\phi_{i+1}\right)$

CU-5: $\quad 3 \frac{\partial \phi_{i-1}}{\partial x}+6 \frac{\partial \phi_{i}}{\partial x}+\frac{\partial \phi_{i+1}}{\partial x}=\frac{1}{3 \Delta x}\left(-\phi_{i-2}-18 \phi_{i-1}+9 \phi_{i}+10 \phi_{i+1}\right)$

Using standard fourier analysis, the numerical approximation to the first derivative becomes $\partial \phi / \partial x=$ $\left[\iota\left(k_{r}^{\prime}-\iota k_{i}^{\prime}\right) / \Delta x\right] \exp (\iota k x / \Delta x)$, where $k_{r}^{\prime}$ and $k_{i}^{\prime}$ are the real and imaginary parts of the modified scaled wavenumber, respectively. The real part $k_{r}^{\prime}$ is associated with dispersion (phase) error, and for the above schemes is given by

UB-1,CD-2: $\quad k_{r}^{\prime}=\sin (k)$

$\mathrm{UB}-3, \mathrm{CD}-4: \quad k_{r}^{\prime}=\frac{4}{3} \sin (k)-\frac{1}{6} \sin (2 k)$

UB-5,CD-6: $\quad k_{r}^{\prime}=\frac{3}{2} \sin (k)-\frac{3}{10} \sin (2 k)+\frac{1}{30} \sin (3 k)$

UB-7,CD-8: $\quad k_{r}^{\prime}=\frac{16}{10} \sin (k)-\frac{4}{10} \sin (2 k)+\frac{8}{105} \sin (3 k)-\frac{1}{140} \sin (4 k)$

$\mathrm{UF}-2, \mathrm{CF}-2: \quad k_{r}^{\prime}=\frac{3}{2} \sin (k)-\frac{1}{4} \sin (2 k)$

CU-5:

$$
k_{r}^{\prime}=\frac{\left(\frac{28}{18} \sin (k)+\frac{1}{18} \sin (2 k)\right)\left(1+\frac{2}{3} \cos (k)\right)+\frac{1}{3} \sin (k)\left(\frac{1}{2}-\frac{8}{18} \cos (k)-\frac{1}{18} \cos (2 k)\right)}{\left(1+\frac{2}{3} \cos (k)\right)^{2}+\left(\frac{1}{3} \sin (k)\right)^{2}}
$$

Note that, as shown by $\mathrm{Li}^{28}{ }^{\prime}$ for each of the UB schemes is equal to that of the CD scheme of the next higher order of accuracy. The imaginary part of the modified scaled wavenumber, $k_{i}^{\prime}$, is associated with 
dissipation error, and is zero for the central difference schemes. For the upwind schemes $k_{i}^{\prime}$ is given by

UB-1: $\quad k_{i}^{\prime}=1-\cos (k)$

UB-3: $\quad k_{i}^{\prime}=\frac{1}{2}-\frac{2}{3} \cos (k)+\frac{1}{6} \cos (2 k)$

UB-5: $\quad k_{i}^{\prime}=\frac{1}{3}-\frac{1}{2} \cos (k)+\frac{2}{10} \cos (2 k)-\frac{1}{30} \cos (3 k)$

UB-7: $\quad k_{i}^{\prime}=\frac{1}{4}-\frac{4}{10} \cos (k)+\frac{2}{10} \cos (2 k)-\frac{6}{105} \cos (3 k)+\frac{1}{140} \cos (4 k)$

$\mathrm{UF}-2: \quad k_{i}^{\prime}=\frac{3}{4}-\cos (k)+\frac{1}{4} \cos (2 k)$

CU-5: $\quad k_{i}^{\prime}=\frac{\left(\frac{1}{2}-\frac{8}{18} \cos (k)-\frac{1}{18} \cos (2 k)\right)\left(1+\frac{2}{3} \cos (k)\right)-\frac{1}{3} \sin (k)\left(\frac{28}{18} \sin (k)+\frac{1}{18} \sin (2 k)\right)}{\left(1+\frac{2}{3} \cos (k)\right)^{2}+\left(\frac{1}{3} \sin (k)\right)^{2}}$

Plots of the real and imaginary parts of the modified wavenumber are shown in figure 1 . In general, the dispersion error (the difference between $k_{r}^{\prime}$ and $k$ ) is reduced for the higher-order schemes. Note, however, that the UF-2 and CF-2 (Fromm-based) schemes, while having only 2nd-order accuracy, have dispersion characteristics comparable to UB-5 and CD-6. The compact upwind CU-5 scheme has superior dispersion characteristics to all others. All of the central difference schemes are free from dissipation error (the difference between $k_{i}^{\prime}$ and zero), while all of the upwind schemes experience an increasing dissipation error at higher wavenumbers. The higher-order UB schemes have a significantly smaller dissipation error than the lowerorder ones. CU-5 has a smaller level of dissipation than UB-7 through most of the wavenumber range, but crosses over to a higher value at the high end of the wavenumber range.

\section{Results and Discussion}

The five central difference and six upwind inviscid flux schemes were tested on three different problems. First, the fourier analysis characteristics just derived were validated using a one-dimensional acoustic standing wave problem. Next, the ability of the schemes to accurately resolve vortex breakdown and transition to turbulence was tested using the Taylor-Green vortex problem. Finally, the performance of the schemes in resolving wall turbulence was tested in a turbulent channel flow calculation.

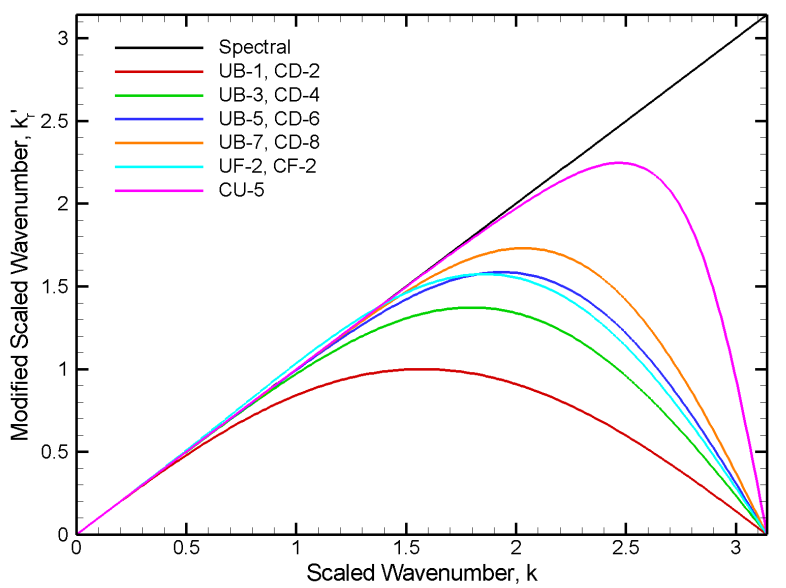

(a) Dispersion Characteristics

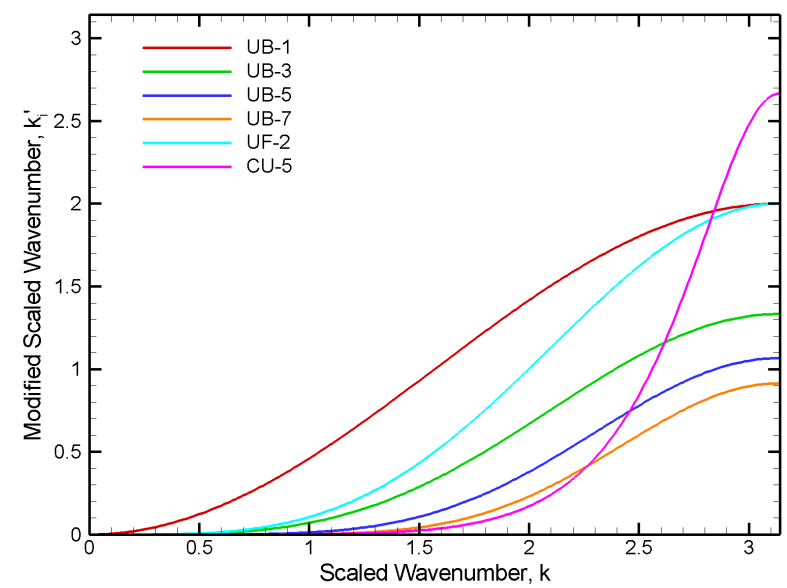

(b) Dissipation Characteristics

Figure 1. Fourier analysis of spatial discretization schemes studied in this work. 


\section{V.A. One-Dimensional Acoustic Standing Wave Problem}

As a simple means of evaluating the ability of the various inviscid flux schemes to resolve high-frequency acoustics or flowfield variations, they were tested on a one-dimensional acoustic standing wave problem. This problem assumes a one-dimensional periodic computational domain in $x$, with $0 \leq x \leq 2 \pi l$, and the reference length $l=1 \mathrm{~m} . N=128$ grid points are used. The domain is initialized with air at $p_{0}=101325 \mathrm{~Pa}, T_{0}=$ $298.15 \mathrm{~K}$, and a small sinusoidal velocity variation as a function of $x$ and specified number of wavelengths, $n: u(x, t=0)=U_{0} \cos (n x / l)$, where $U_{0}=0.1 \mathrm{~m} / \mathrm{s}$. Under the assumption of isentropic, small disturbance inviscid flow, the Navier-Stokes equations can be simplified to the linearized equations of gas dynamics, and an exact solution derived as

$$
\begin{array}{r}
u(x, t)=\frac{1}{2} U_{0}\left[\cos \left(\frac{n\left(x-a_{0} t\right)}{l}\right)+\cos \left(\frac{n\left(x+a_{0} t\right)}{l}\right)\right]=U_{0} \cos \left(\frac{n x}{l}\right) \cos \left(\frac{n a_{0} t}{l}\right) \\
p(x, t)-p_{0}=\frac{1}{2} \rho_{0} a_{0} U_{0}\left[\cos \left(\frac{n\left(x-a_{0} t\right)}{l}\right)-\cos \left(\frac{n\left(x+a_{0} t\right)}{l}\right)\right]=\rho_{0} a_{0} U_{0} \sin \left(\frac{n x}{l}\right) \sin \left(\frac{n a_{0} t}{l}\right)
\end{array}
$$

where $t$ is time, $\rho_{0}$ is the initial density $\left(1.18413 \mathrm{Kg} / \mathrm{m}^{3}\right)$, and $a_{0}$ is the initial sound speed $(346.117 \mathrm{~m} / \mathrm{s})$.

Simulations of this problem were carried out for all eleven inviscid flux schemes for six different numbers of initial wavelengths in the domain. Initial numbers of wavelengths of $n=1,2,4,8,16$ and 32 , corresponding to $128,64,32,16,8$ and 4 grid points per wavelength (PPW), respectively, were tested. The corresponding scaled wavenumbers are $k=2 \pi n / N=\pi / 64, \pi / 32, \pi / 16, \pi / 8, \pi / 4$ and $\pi / 2$. The simulations for $n=1,2,4$ and 8 were run at a Courant-Friedrich-Lewy number (CFL) number of unity: $\Delta t=2 \pi l /\left(N a_{0}\right)=1.41823 \times$ $10^{-4} \mathrm{~s}$. However, due to concern that this timestep was too large to adequately resolve the acoustics for the $n=16$ and $n=32$ cases, they were run at a timestep equal to $1 / 16$ of the characteristic acoustic timescale of the problem, $2 \pi l /\left(n a_{0}\right)$, resulting in $\Delta t=7.09116 \times 10^{-5} \mathrm{~s}$ and $\Delta t=3.54558 \times 10^{-5} \mathrm{~s}(\mathrm{CFL}$ numbers of 0.5 and 0.25 ), respectively. It is worthwhile to point out that, for this simple acoustic problem, the divergence form central difference schemes also ran successfully, and produced results identical to the baseline skew-symmetric central difference schemes.

The results for $n=1,2$ and 4 are not shown in this paper simply because there is little difference between all the schemes in those cases, with the sole exception of UB-1, which exhibits noticeable dissipation even at $n=1$. However, greater differences between the schemes become evident at $n=8,16$ and 32 . The pressure perturbation time histories at the first pressure antinode in the domain are shown for these cases in figure 2. The left column shows the results for the central schemes, while the right column shows the results for the upwind schemes. The calculations were run through 10 acoustic timescales for each case. However, for clarity, only the results for the first two acoustic timescales are shown.

As expected, there is no dissipation present in all of the central difference schemes, though the dispersion error of the CD-2 scheme, noticeable at $n=8$, becomes more pronounced at $n=16$, and severe by $n=32$. The dispersion error of the CD- 4 scheme is also pronounced at $n=32$, and the error is noticeable for the other central schemes as well. Interestingly, the CF-2 scheme has a dispersion error in the opposite direction from the other schemes at $n=8$ and $n=16$, consistent with the dispersion characteristics shown in figure 1a.

The dissipative characteristics of the upwind schemes are clearly visible in the right column of figure 2 . The UB- 1 scheme is severely dissipative for $n=8,16$ and 32 . Dissipation for UF-2 and UB-3 is just noticeable at $n=8$, more pronounced at $n=16$, and severe by $n=32$. The dissipation present in UB- 5 , UB-7 and CU-5 only becomes significant at $n=32$, with UB-7 less dissipative than UB-5, and CU-5 even better. Consistent with figure 1a, the CU-5 scheme displays very little dispersion error, even at $n=32$.

Eqs. (47) and (48) can be modified to account for the effect of dispersion and dissipation discussed in the fourier analysis section, resulting in

$$
\begin{gathered}
u(x, t)=U_{0} \cos \left(\frac{n x}{l}\right) \cos \left(\frac{k_{r}^{\prime}}{k} \frac{n a_{0} t}{l}\right) \exp \left(-\frac{k_{i}^{\prime}}{k} n a_{0} t\right) \\
p(x, t)-p_{0}=\rho_{0} a_{0} U_{0} \sin \left(\frac{n x}{l}\right) \sin \left(\frac{k_{r}^{\prime}}{k} \frac{n a_{0} t}{l}\right) \exp \left(-\frac{k_{i}^{\prime}}{k} n a_{0} t\right)
\end{gathered}
$$

where $k$ is scaled from $n$, and $k_{r}^{\prime}$ and $k_{i}^{\prime}$ are obtained for the numerical scheme under consideration from Eqs. (35)-(46). There is excellent agreement between the numerical calculations and these modified equations, suggesting that the timestep and time-advancement scheme were sufficiently accurate to resolve both the acoustics and the numerical effects from each scheme. 


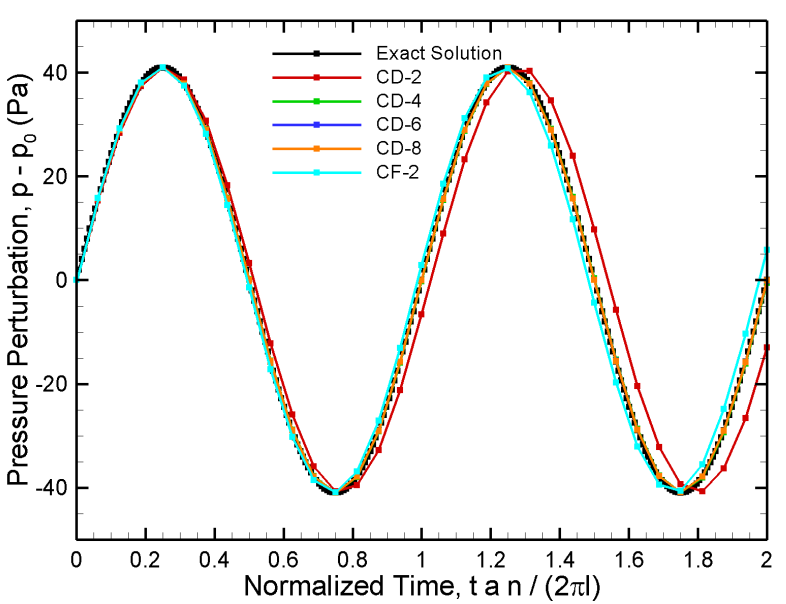

(a) $n=8,16 \mathrm{PPW}, k=\pi / 8$

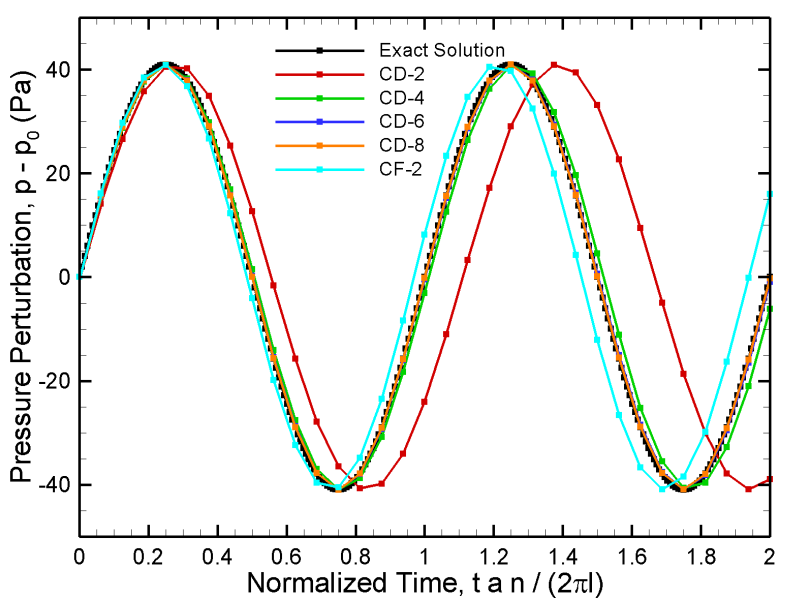

(c) $n=16,8 \mathrm{PPW}, k=\pi / 4$

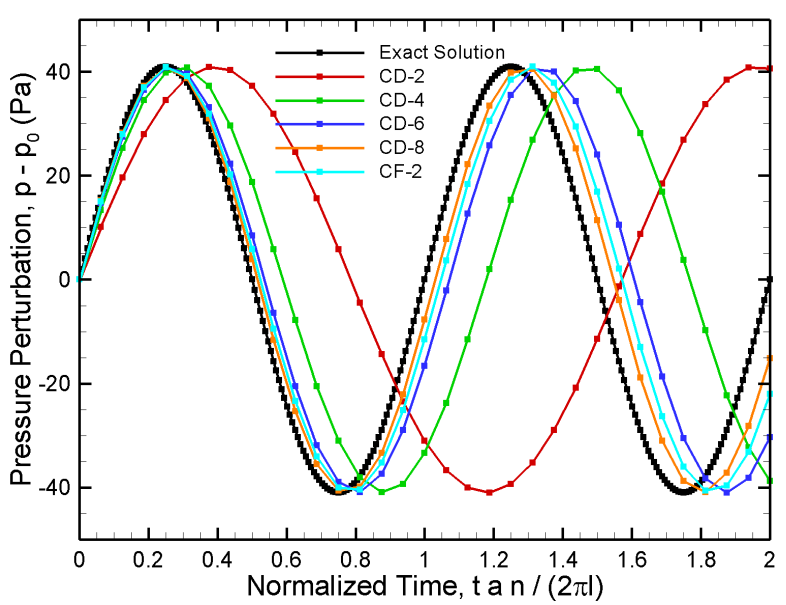

(e) $n=32,4 \mathrm{PPW}, k=\pi / 2$

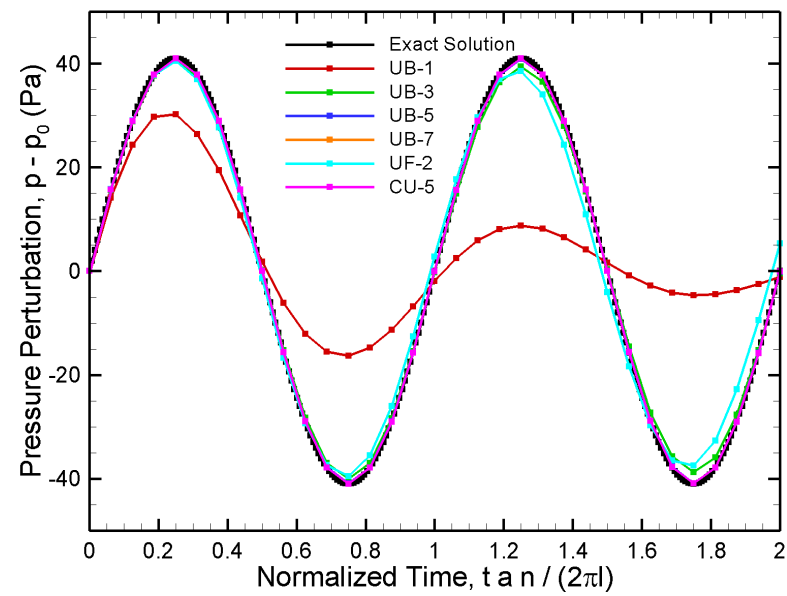

(b) $n=8,16 \mathrm{PPW}, k=\pi / 8$

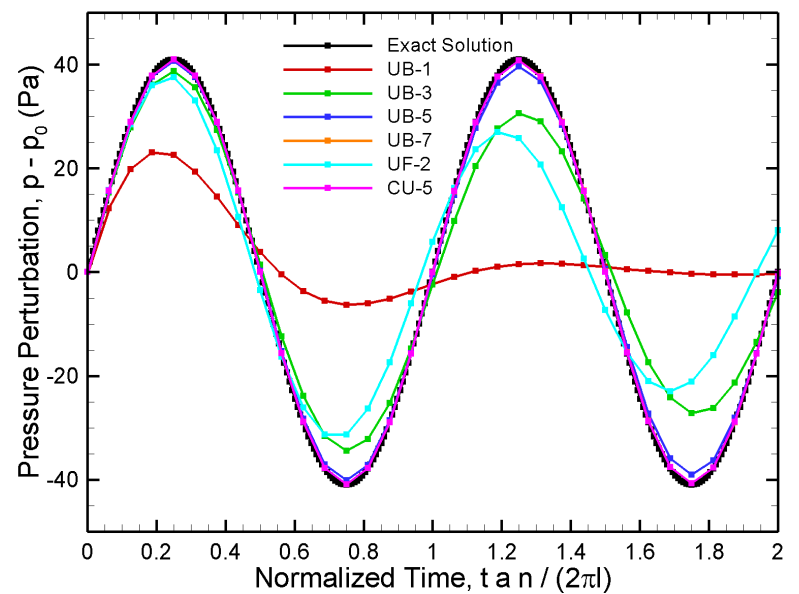

(d) $n=16,8$ PPW, $k=\pi / 4$

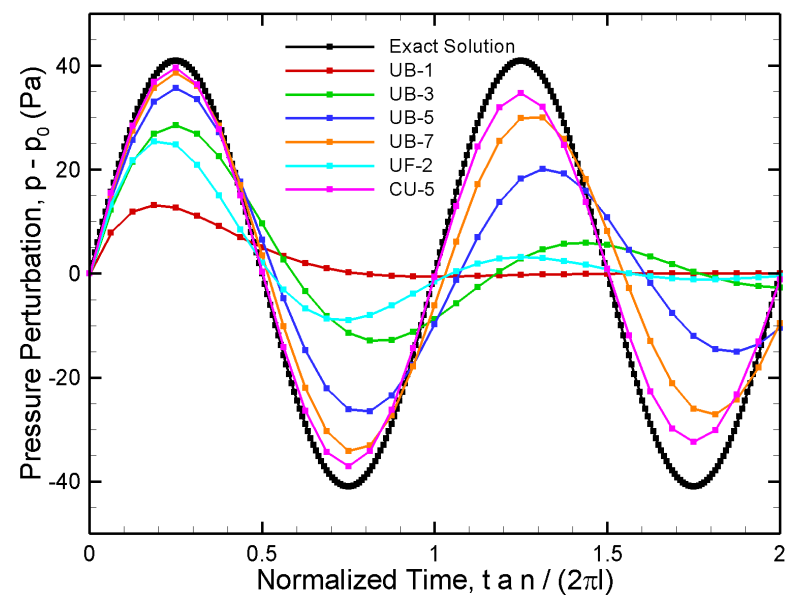

(f) $n=32,4 \mathrm{PPW}, k=\pi / 2$

Figure 2. Comparison of pressure time histories at the first pressure antinode in the acoustic standing wave problem. One-dimensional grid is $\mathbf{1 2 8}$ points. Note that PPW stands for grid points per wavelength. Left column: central schemes. Right column: upwind schemes

\section{V.B. Taylor-Green Vortex Problem}

The Taylor-Green vortex problem is a benchmark case which, from a smooth initial condition, simulates vortex stretching and transition to turbulence. It was used as a test case for the $1^{\text {st }}$ International Workshop 
on High-Order CFD Methods, held at the $50^{\text {th }}$ AIAA Aerospace Sciences Meeting, January 7-8, 2012, in Nashville, Tennessee (http://www.public.iastate.edu/ zjw/hiocfd.html). DNS results have been calculated by Brachet et al. ${ }^{29}$ and, more recently by van Rees et al. ${ }^{30}$ The latter results are used as the basis for comparison in this paper.

The solution is computed on a periodic cube domain, $-\pi l \leq x, y, z \leq \pi l$. The flowfield is initialized with

$$
\begin{aligned}
u & =U_{0} \sin \left(\frac{x}{l}\right) \cos \left(\frac{y}{l}\right) \cos \left(\frac{z}{l}\right) \\
v & =-U_{0} \cos \left(\frac{x}{l}\right) \sin \left(\frac{y}{l}\right) \cos \left(\frac{z}{l}\right) \\
w & =0 \\
p & =p_{0}+\frac{\rho_{0} U_{0}^{2}}{16}\left[\cos \left(\frac{2 x}{l}\right)+\cos \left(\frac{2 y}{l}\right)\right]\left[\cos \left(\frac{2 z}{l}\right)+2\right]
\end{aligned}
$$

where here $l=0.01 \mathrm{~m}, p_{0}=7271 \mathrm{~Pa}, T_{0}=298.15 \mathrm{~K}$ and $U_{0}=34.6115 \mathrm{~m} / \mathrm{s}$. The sound speed $a_{0}=$ $346.115 \mathrm{~m} / \mathrm{s}$. The flow is effectively incompressible, $M_{0}=U_{0} / a_{0}=0.1$. The initial condition for $\rho$ is calculated by the perfect gas law, assuming fixed $T=T_{0}$. The transport properties are fixed at their values at $T_{0}$ throughout the calculation. The resultant Reynolds number is $R e=\rho_{0} U_{0} l / \mu_{0}=1600$. Calculations for each scheme were performed on three grid sizes: $256 \times 256 \times 256,192 \times 192 \times 192$ and $128 \times 128 \times 128$ grid points, with uniform grid spacing throughout the domain. The characteristic timescale of the problem is $l / U_{0}=2.8892 \times 10^{-4} \mathrm{~s}$. The calculations were run using a constant timestep of 0.001 times this timescale, $2.8892 \times 10^{-7} \mathrm{~s}$, which even on the $256 \times 256 \times 256$ grid is well below an approximate CFL characteristic timescale of $\Delta x /\left(U_{0}+a_{0}\right)=6.4465 \times 10^{-7} \mathrm{~s}(\mathrm{CFL} \approx 0.45)$. The calculations were run to 20 characteristic timescales.

It should be mentioned that while the baseline skew-symmetric central difference schemes ran successfully to completion on all cases, simulations using divergence form central difference schemes typically failed shortly before a normalized time of $t^{*}=t U_{0} / l=4$. Baseline central difference simulations were also run on a $64 \times 64 \times 64$ point grid with the viscous terms removed from the equations. These simulations confirmed the kinetic energy conservation property of these schemes.

The time evolution of the Taylor-Green vortex flowfield can be visualized by showing iso-surfaces of the Q-criterion ${ }^{31}$ at various times (figure 3 ). $Q>0$ indicates regions of a flowfield in which vorticity dominates over strain. Here, iso-surfaces of $Q=0.1\left(U_{0} / l\right)^{2}=1.19797 \times 10^{6} \mathrm{~s}^{-2}$ are plotted. This calculation was performed using the CD- 8 scheme on the $256 \times 256 \times 256$ grid. Time is normalized by the characteristic timescale, $t^{*}=t U_{0} / l$, and the iso-surfaces are colored with the normalized velocity magnitude, $U / U_{0}$. It is evident from the figure that the initial large-scale vortex structure at $t^{*}=0$ steadily evolves toward smaller vortex structures $\left(t^{*}=4\right)$. By $t^{*}=8$, the flow has transitioned to turbulence, and proceeds steadily to a more isotropic state. The symmetries in the flowfield, discussed by Ref. 29, are evident from figure 3. Also note that the reduction in overall kinetic energy as the simulation progresses is visible in the velocity magnitude color scale .

Time histories for the mean kinetic energy, measured mean kinetic energy dissipation rate, and mean enstrophy for the central difference and upwind schemes on the $256 \times 256 \times 256$ point grid are compared with the spectral DNS (which was calculated on a $512 \times 512 \times 512$ point grid) of Ref. 30 in figure 4 . The mean kinetic energy for the domain is given by

$$
E_{k, m}=\frac{1}{\rho_{0} V} \int \rho \frac{U^{2}}{2} d V
$$

The mean measured kinetic energy dissipation rate is $\varepsilon=-\partial E_{k, m} / \partial t$. This value was calculated from the mean kinetic energy history using standard $2^{\text {nd }}$ order central differences for all but the first and last time history points (which used one-sided differences).

The mean enstrophy for the domain is given by

$$
\Omega_{m}=\frac{1}{\rho_{0} V} \int \rho \frac{\boldsymbol{\omega} \cdot \boldsymbol{\omega}}{2} d V
$$

where the vorticity vector $w$ at each point in the domain was calculated using $8^{\text {th }}$ order central differences (i.e. Eq. (27) is used for determining the velocity derivative components of the vorticity vector). It is 


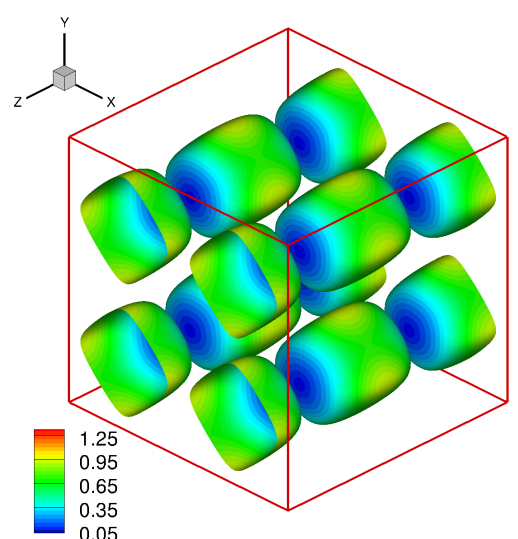

(a) $\mathrm{t}^{*}=0$

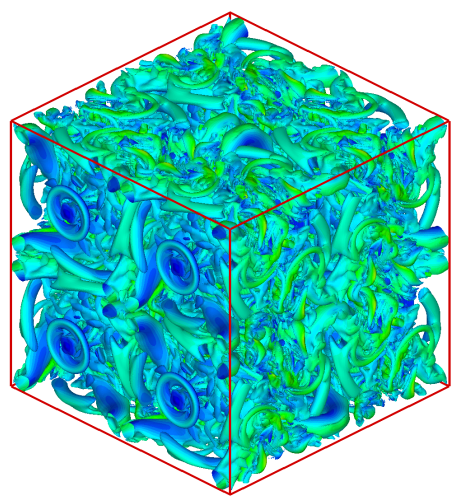

(d) $\mathrm{t}^{*}=12$

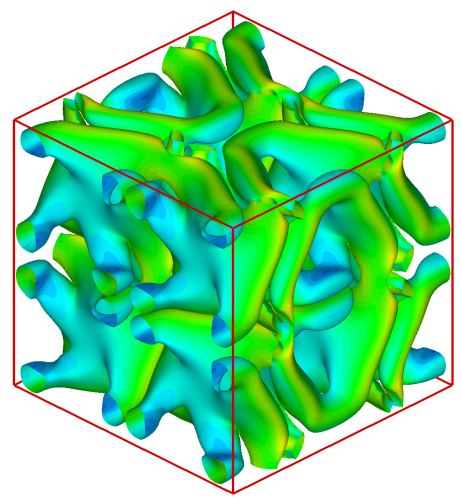

(b) $\mathrm{t}^{*}=4$

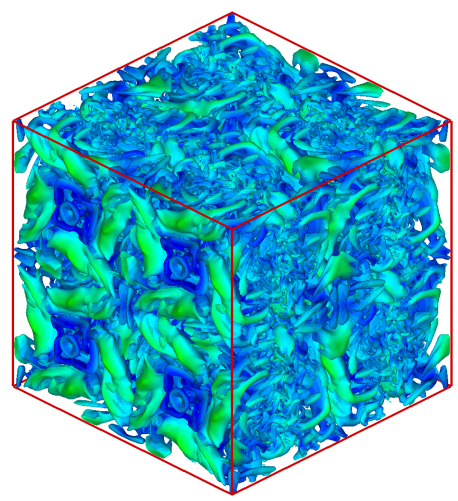

(e) $\mathrm{t}^{*}=16$

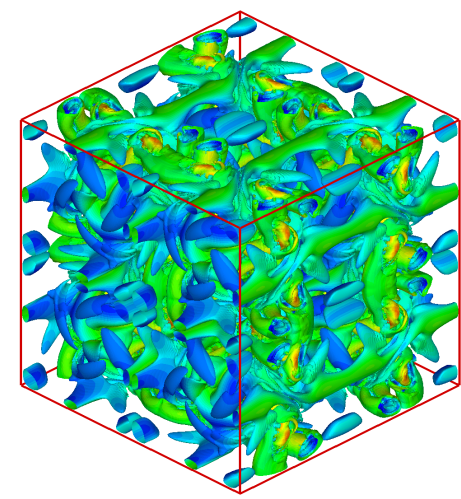

(c) $\mathrm{t}^{*}=8$

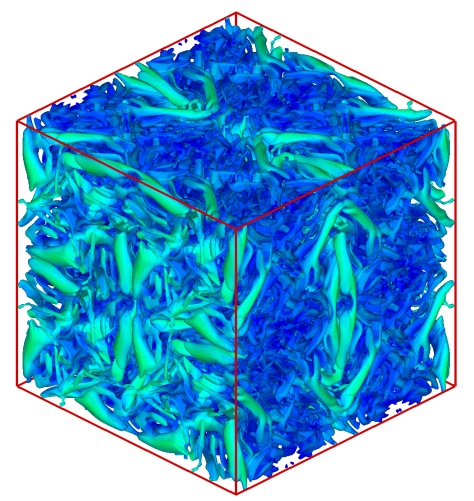

(f) $\mathrm{t}^{*}=20$

Figure 3. Iso-surfaces of Q-criterion $Q=0.1\left(U_{0} / l\right)^{2}$ showing the time evolution of the Taylor-Green vortex problem at $R e=1600$, computed with the CD-8 scheme on a $256 \times 256 \times 256$ point grid. Iso-surfaces are colored with the normalized velocity magnitude, $U / U_{0} \cdot t^{*}$ is a normalized timescale, $t U_{0} / l$

arguable whether this methodology is appropriate for the lower-order flux schemes. It does, however, lead to a consistent comparison across all schemes.

It is evident from figure 4 that the central difference schemes at this grid resolution are in very good agreement with the reference DNS results for all three mean flow properties. The CD-2 scheme, which like all central schemes is non-dissipative, but has limited range of phase accuracy, does exhibit worse agreement with respect to $\varepsilon$ and $\Omega_{m}$ than the other central schemes near the peak of dissipation at $t^{*}=9$, though it is still reasonably accurate. There are greater differences visible for the upwind schemes. Considering the mean kinetic energy history first, the UB-1 scheme, not surprisingly, rapidly dissipates all kinetic energy in the flow. The UF-2 and UB-3 schemes follow the reference $E_{k, m}$ time history reasonably well, although some differences from the reference DNS are visible, while UB-5, UB-7 and CU-5 are in very good agreement. With respect to the measured dissipation rate, $\varepsilon$, UB-5, UB-7 and CU-5 again agree very well with the reference DNS, while greater differences are evident for UF-2 and UB-3. Note that UF-2 and UB-3 have a lower dissipation peak that occurs earlier in time (at $t^{*}=8.45$ ). The measured $\varepsilon$ for UB-1 was so large in comparison to the others that for clarity it is not shown in figure 4. The upwind schemes exhibit the largest differences from the reference DNS in the mean enstrophy history, $\Omega_{m}$. Again, as expected, UB-1 rapidly dissipates all enstrophy in the flow. None of the other upwind schemes match the reference DNS, though UB-7 and CU-5 do the best and UF-2 performs the worst here. These results are consistent with the dissipative characteristics shown in figure $1 \mathrm{~b}$.

It is noteworthy that UB-5, UB-7 and CU-5, which still miss the $\Omega_{m}$ history near peak dissipation at $t^{*}=9$, still match the overall history of $E_{k, m}$ and $\varepsilon$ so well. For an incompressible flow, the theoretical value of $\varepsilon$ is directly proportional to $\Omega_{m}, \varepsilon=2 \mu \Omega_{m} / \rho_{0}$. Though compressibility effects are present in this calculation, their magnitude is small at this grid resolution. Therefore, the good agreement of UB-5, UB-7 and CU-5 

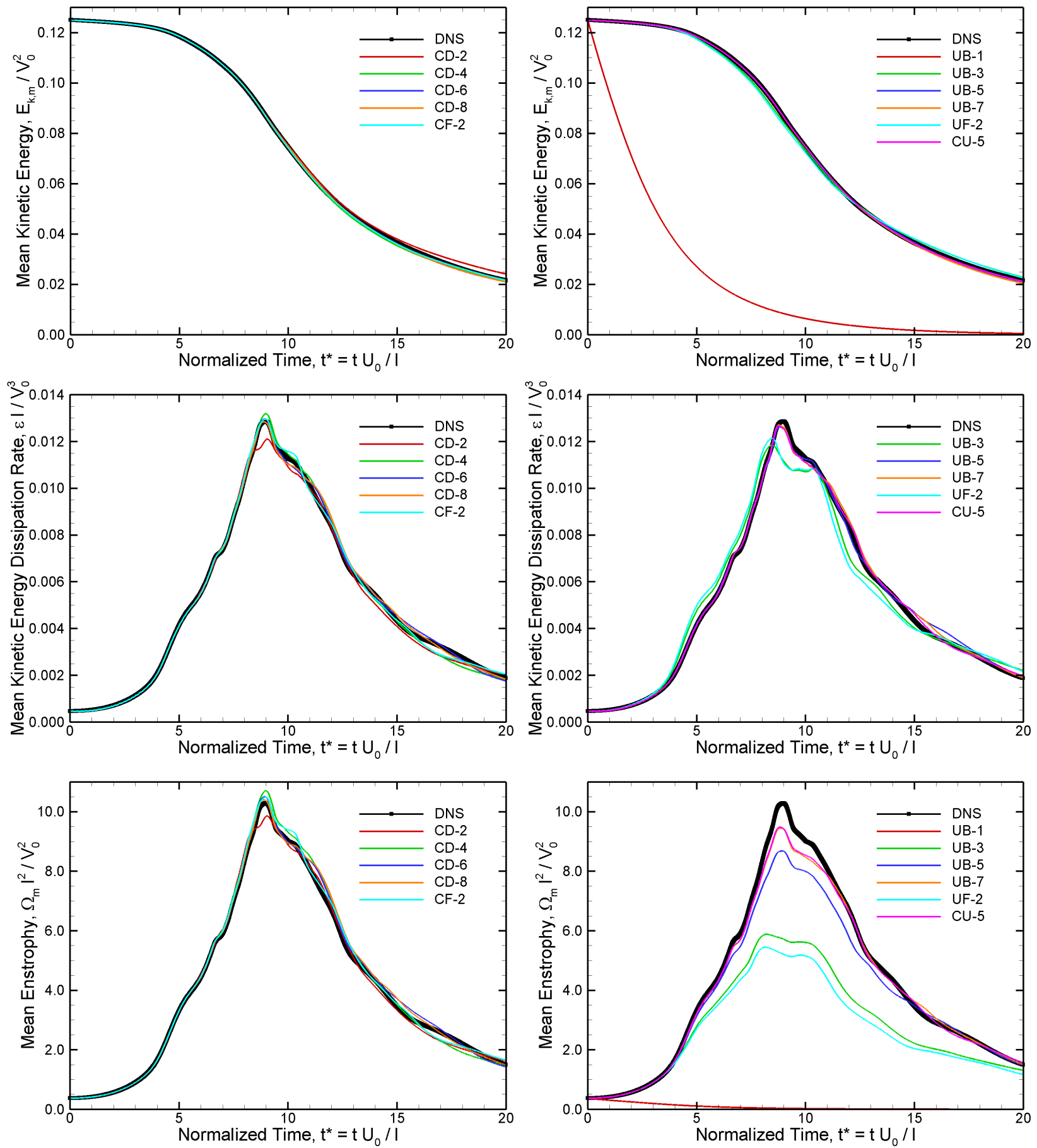

Figure 4. Plots showing the time evolution of the normalized mean kinetic energy, $E_{k, m} / V_{0}^{2}$ (upper panel), normalized measured mean kinetic energy dissipation rate, $\varepsilon l / V_{0}^{3}$ (middle panel), and normalized mean enstrophy, $\Omega l^{2} / V_{0}^{2}(\mathrm{bottom}$ panel) for the reference spectral DNS on a $512 \times 512 \times 512$ point grid, and the central and upwind schemes schemes on $256 \times 256 \times 256$ point grids. Left column: central schemes. Right column: upwind schemes.

with the dissipation history suggests that, at this grid resolution, the numerical dissipation exhibited by the upwind schemes at the small flow length scales is largely making up for the physical dissipation captured by the DNS and central schemes at those scales. Note that even UF-2 and UB-3, with much greater divergences from the $\Omega_{m}$ history, follow the reference $E_{k, m}$ time history reasonably well (though $\varepsilon$ not as well).

The dissipative characteristics of the lower-order upwind schemes are plainly visible in figure 5 , where iso-contours of normalized vorticity magnitude $\|\boldsymbol{\omega}\| l / U_{0}$ are shown on a section of the $x=0$ plane at $t^{*}=8$. 


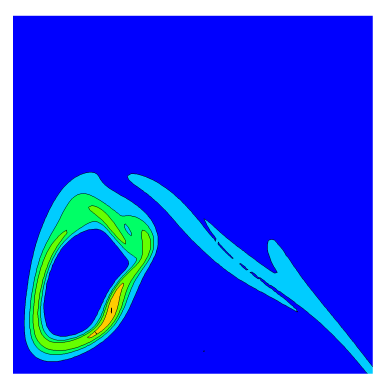

(a) DNS

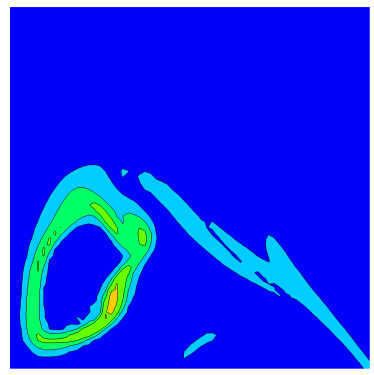

(e) CD-8

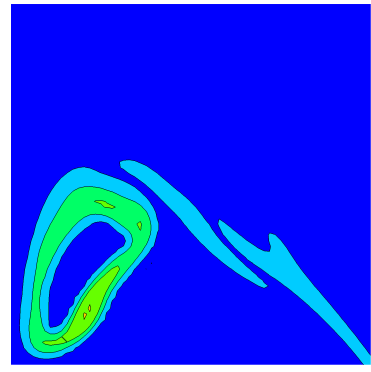

(i) UB-5

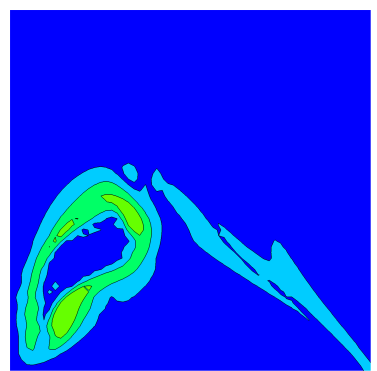

(b) CD-2

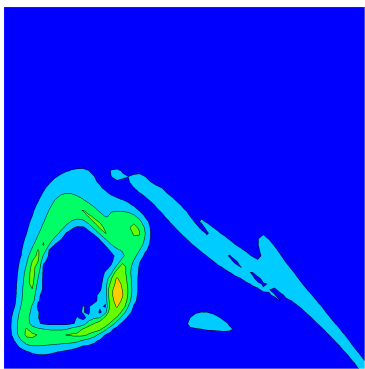

(f) $\mathrm{CF}-2$

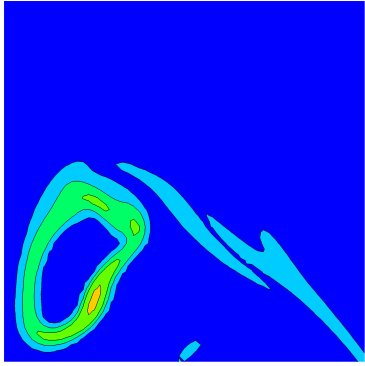

(j) UB-7

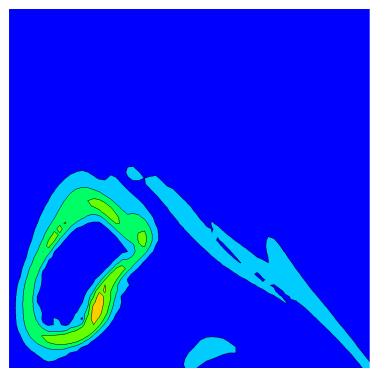

(c) CD-4

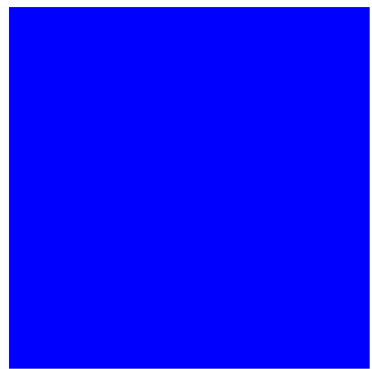

(g) UB-1

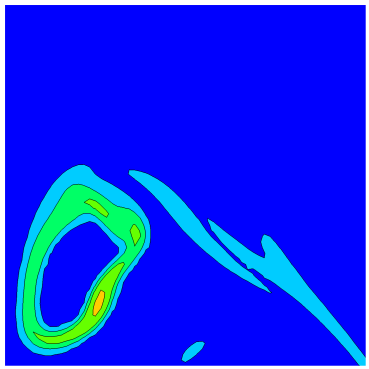

(k) CU-5

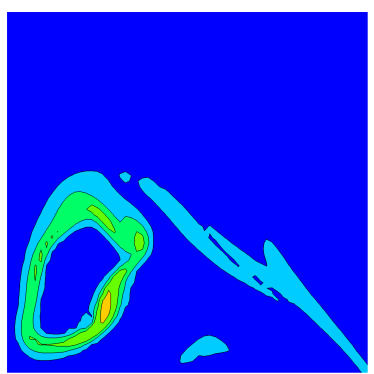

(d) CD-6

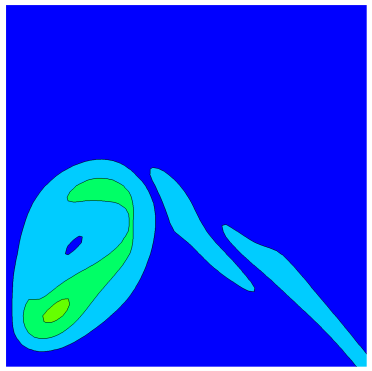

(h) UB-3

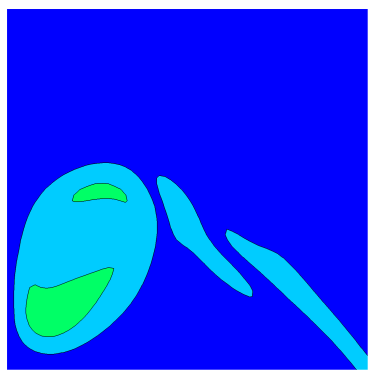

(l) UF-2

Figure 5. Comparison of iso-contours of normalized vorticity magnitude $\|\omega\| l / U_{0}$ on a section of the $x=0$ plane for all schemes at $t^{*}=8$ on a $256 \times 256 \times 256$ point grid. Contour levels are 1, 5, 10, 20 and 30 . Note that the DNS was performed on a $512 \times 512 \times 512$ point grid.

All plots, except for the reference DNS, are extracted from the $256 \times 256 \times 256$ grid solutions. The portion of the $x=0$ plane shown is from $-\pi l \leq y-\pi l / 2$ (shown as the $x$-axis in the plots) and $\pi l / 2 \leq z \leq \pi l$ (shown as the $y$-axis in the plots). The contour levels shown are 1, 5, 10, 20 and 30. UB-1 has completely dissipated the vorticity on this plane by $t^{*}=8$, while UF-2 and UB-3 show significant smoothing of the vorticity compared to the DNS reference. The higher-order upwind schemes and the central schemes all show generally good agreement with the DNS, though again CD-2 does not resolve the vortex structure as sharply as the other central schemes.

For comparison, time histories for $E_{k, m}$, measured $\varepsilon$, and $\Omega_{m}$ for the central difference and upwind schemes on the $128 \times 128 \times 128$ point grid are compared with the spectral DNS in figure 6 . At this lower grid resolution, the central schemes still do reasonably well at matching the DNS, though there is a greater deviation in $\varepsilon$ and $\Omega_{m}$ than was present on the $256 \times 256 \times 256$ point grid. The CD-2 scheme now exhibits an earlier peak to these properties (at $t^{*}=8.62$ ) than the other central schemes. Unsurprisingly, the upwind schemes UF-2 and UB-3 show very significant differences compared to the reference DNS. From $t^{*}=0-8$, $\Omega_{m}$ for these schemes is much lower than the DNS history, while the measured $\varepsilon$ is much higher, suggesting that the numerical dissipation in these schemes is too large, and resulting in the faster decay of $E_{k, m}$ than the DNS. UB-5, UB-7 and CU-5 again do significantly better, and match the $E_{k, m}$ history reasonably well.

As was mentioned above, results for all schemes at a grid resolution of $192 \times 192 \times 192$ were also calculated. 

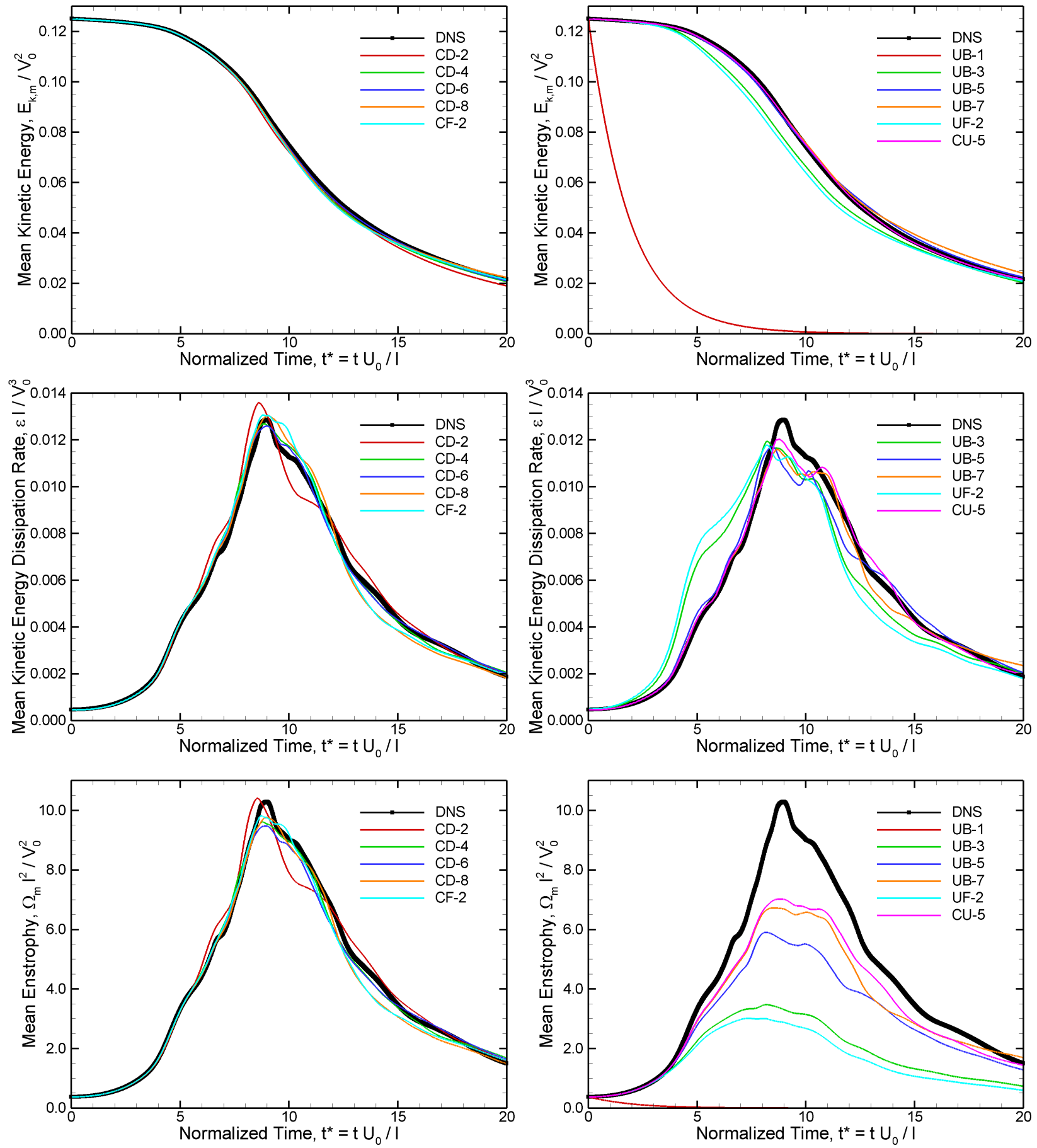

Figure 6. Plots showing the time evolution of the normalized mean kinetic energy, $E_{k, m} / V_{0}^{2}$ (upper panel), normalized measured mean kinetic energy dissipation rate, $\varepsilon l / V_{0}^{3}$ (middle panel), and normalized mean enstrophy, $\Omega l^{2} / V_{0}^{2}$ (bottom panel) for the reference spectral DNS on a $512 \times 512 \times 512$ point grid, and the central and upwind schemes schemes on $128 \times 128 \times 128$ point grids. Left column: central schemes. Right column: upwind schemes.

The results are intermediate between the larger and smaller grid resolutions.

\section{V.C. Turbulent Channel Flow Problem}

Turbulent channel flow has been extensively studied by experiments ${ }^{32}$ and DNS studies, ${ }^{33,34}$ and the mean flow profile and statistics have been very well characterized. Therefore, it is an excellent benchmark case 
for evaluating various inviscid flux schemes for DNS or LES of wall-bounded shear flows. Here, turbulent channel flow of air at $p_{0}=25331 \mathrm{~Pa}, T_{0}=298.15 \mathrm{~K}$ and a mean streamwise velocity $u_{m}=44.44 \mathrm{~m} / \mathrm{s}$ is simulated. The flow is essentially incompressible $\left(M_{m}=0.127\right)$. The computational domain is $0 \leq x \leq 2 \pi h$ in the streamwise $(x-)$ direction, $-h \leq y \leq h$ in the vertical ( $y$-) direction, and $0 \leq z \leq \pi h$ in the spanwise $\left(z\right.$-) direction. The channel half-width, $h$, is $0.01 \mathrm{~m}$. The Reynolds number based on $u_{m}$ and the full channel width, $2 h$, is $R e_{2 h}=\rho_{m} u_{m} 2 h / \mu_{m}=14300$. The DNS calculation in Ref. 34 was run at Reynolds number based on wall variables $R e_{\tau}=\rho_{w} u_{\tau} h / \mu_{w}=395$, which is equivalent to $R e_{2 h}=13800$, about $4 \%$ less than this calculation. Note that the wall friction velocity $u_{\tau}=\sqrt{\tau_{x y, w} / \rho_{w}}$.

Periodic boundary conditions are used in both the $x$ - and $z$-directions. Adiabatic, no-slip wall boundary conditions are imposed on the bottom and top $(y-)$ surfaces. In order to prevent the viscous losses at both walls from slowly "wearing down" the flow, the integrated area-sum of these forces is computed at each time step, and added in as a body force term, $f_{x}$, uniformly over the entire computational volume $\left(f_{y}\right.$ and $f_{z}$ are zero). This term effectively reproduces the effect of the pressure gradient needed to sustain a real turbulent channel flow, and maintains a constant mass flow rate $\left(\dot{m}=8.266 \times 10^{-3} \mathrm{~kg} / \mathrm{s}\right)$ in the simulation.

Simulations for all schemes were run on four different grid sizes: $128 \times 129 \times 128,96 \times 129 \times 96,64 \times 129 \times 64$ and $48 \times 129 \times 48$ grid points. The grid spacing is uniform in $x$ and $z$, and stretched by the hyperbolic tangent function in $y$. The wall-normal grid spacing at the walls is $0.001 \mathrm{~h}$. The $128 \times 129 \times 128$ grid is at a near-DNS level of resolution for this flow (the DNS of Ref. 34 was run on a $256 \times 257 \times 256$ point grid). The grids with progressively coarser spacing in $x$ and $z$ were run in order to assess the sensitivity of the results to grid spacing in those directions. The grid size in the $y$-direction was held constant because of the sensitivity of the central difference schemes to grid stretching. The maximum grid stretch rate $\left(y_{j+1}-y_{j}\right) /\left(y_{j}-y_{j-1}\right)$ with 129 points in the $y$-direction is 1.081 , and all of the central schemes were stable at this stretch rate. With 97 points in the $y$-direction the maximum stretch rate is 1.118, which is stable for the CD-2 scheme, but not for the other central schemes. None of the central schemes were stable with 65 points in the $y$-direction (maximum stretch rate of 1.201). One additional simulation was run on a $192 \times 193 \times 192$ point grid using the CD-2 scheme (maximum stretch rate of 1.047).

The simulations for all the central schemes on all grids were run using a fixed timestep of $7.0685 \times 10^{-8} \mathrm{~s}$, which corresponds to an approximate CFL number of 2.45, based on the wall-normal spacing of $0.001 \mathrm{~h}$ at the walls and a sound speed $a_{0}=346.115 \mathrm{~m} / \mathrm{s}$. The UB-3, UB-5 and UB-7 upwind schemes were also stable at this timestep. However, the UB-1, CF-2 and CU-5 schemes were generally not stable and most of their cases were run at a fixed timestep $25 \%$ smaller, $5.6548 \times 10^{-8} \mathrm{~s}$ (the exceptions being UB- 1 on the $128 \times 129 \times 128$, which for stability was run at an even smaller timestep of $4.4178125 \times 10^{-8} \mathrm{~s}$, and CF- 2 on the $48 \times 129 \times 48$ grid which was stable at the original timestep). All of these timesteps are more than two orders of magnitude smaller than the characteristic timescale of near-wall turbulence, which from Choi and Moin ${ }^{35}$ is $\mu_{w} /\left(\rho_{w} u_{\tau}^{2}\right) \simeq 1.0 \times 10^{-5} \mathrm{~s}$ for a well-resolved simulation of this problem. Obviously, the explicit Runge-Kutta time-advancement scheme, which is limited in stability by the fine near-wall grid spacing, is likely not optimally efficient for this problem.

The flow is initialized using a power law mean velocity profile, along with unit wavelength $(n=1)$ oscillations in $y$ and $z$, and $n=2$ oscillations in $x$. This divergence-free initial condition was borrowed from Moin and $\mathrm{Kim}^{36}$

$$
\begin{aligned}
& u=u_{c, 0}\left[1-\left(\frac{y}{h}\right)^{8}\right]+U_{0} \pi \cos \left(\frac{2 x}{h}\right) \sin \left(\frac{\pi y}{h}\right) \sin \left(\frac{2 z}{h}\right) \\
& v=-U_{0} \sin \left(\frac{2 x}{h}\right)\left[1+\cos \left(\frac{\pi y}{h}\right)\right] \sin \left(\frac{2 z}{h}\right) \\
& w=-U_{0} \frac{\pi}{2} \sin \left(\frac{2 x}{h}\right) \sin \left(\frac{\pi y}{h}\right) \cos \left(\frac{2 z}{h}\right)
\end{aligned}
$$

where here the initial centerline streamwise velocity, $u_{c, 0}=50 \mathrm{~m} / \mathrm{s}$ and $U_{0}=5 \mathrm{~m} / \mathrm{s}$.

This initial condition, as well as a fully developed turbulent channel flow, can be visualized by showing iso-surfaces of $Q=0.1\left(u_{m} / h\right)^{2}=1.97491 \times 10^{6} \mathrm{~s}^{-2}$ at $t^{*}=0$ and $t *=30$ (figure 7). Again, $Q>0$ indicates regions of a flowfield in which vorticity dominates over strain. $\mathrm{t}^{*}$ is a normalized timescale, $t^{*}=t u_{m} / 2 \pi h$, representing the number of streamwise flowthrough times. The iso-surfaces are colored with the normalized velocity magnitude, $U / u_{m}$. This calculation was performed using the CD- 8 scheme on a $128 \times 129 \times 128$ point grid. Further details of this case follow below. 


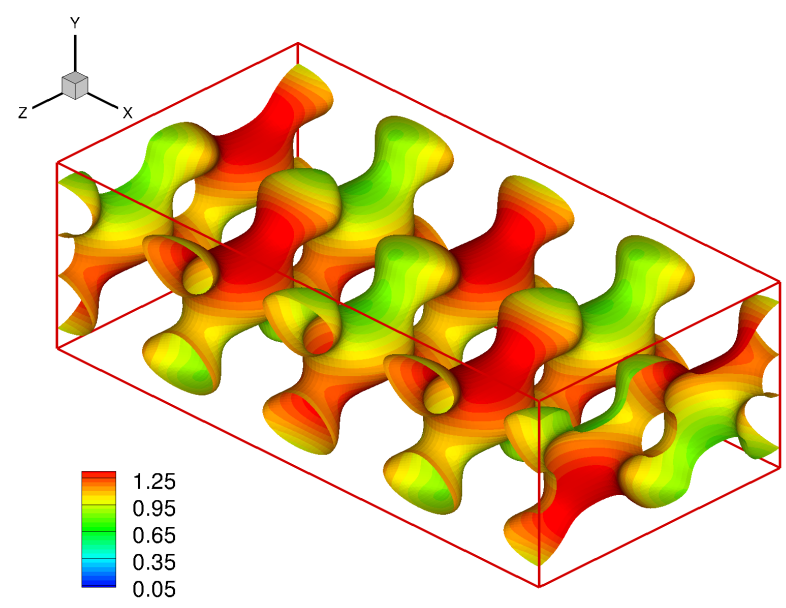

(a) $t^{*}=0$

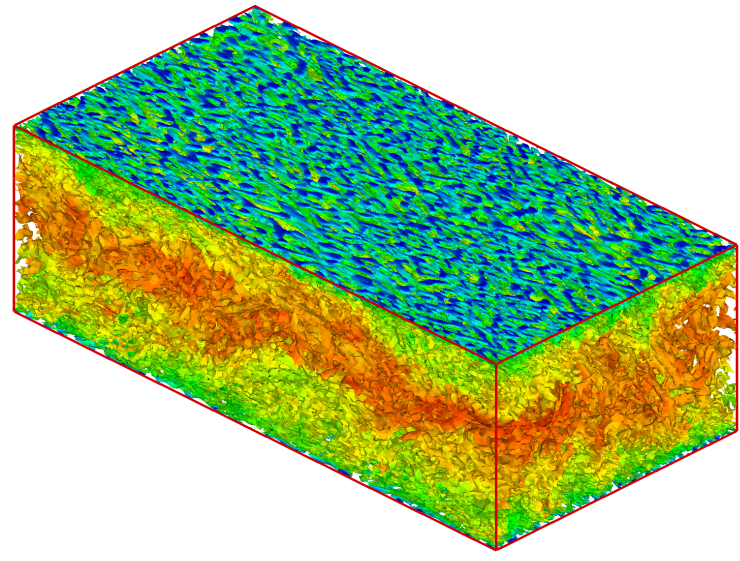

(b) $\mathrm{t}^{*}=30$

Figure 7. Iso-surfaces of Q-criterion $Q=0.1\left(u_{m} / h\right)^{2}$ showing turbulent channel flow at $R e=14300$, computed with the CD-8 scheme on a $128 \times 129 \times 128$ point grid. a) initial condition, b) statistically stationary state. Iso-surfaces are colored with the normalized velocity magnitude, $U / u_{m}$. $\mathrm{t}^{*}$ is a normalized timescale, $t^{*}=t u_{m} / 2 \pi h$, representing the number of streamwise flowthrough times.

The central difference cases were started from the initial condition, and it was evident from the skin friction history that the flow rapidly transitioned to turbulence within the first few flowthrough times. Based on the integrated boundary flux values on the viscous walls, the flowfields were statistically stationary by $t^{*}=15$. Individual flowfield states were then saved every tenth of a flowthrough time from $t^{*}=15-30$ (151 individual flowfield states were saved for each case). These flowfields were then time-averaged, as well as space-averaged in the periodic directions $x$ and $z$. For comparison, an additional averaging process was also performed in which the two halves of the channel $(-h \leq y \leq 0$ and $0 \leq y \leq h)$ were also averaged together. Due to the adiabatic walls, the mean temperature and pressure increased by $0.28 \%$ over $t^{*}=15-30$.

The upwind scheme cases on the $128 \times 129 \times 128$ and $96 \times 129 \times 96$ point grids were started from the corresponding CD-2 flowfield state saved at $t^{*}=10$, while the upwind cases on the $64 \times 129 \times 64$ and $48 \times 129 \times 48$ point grids were started from the corresponding CD- 2 states at $t^{*}=12$ and $t^{*}=15$, respectively. The higher-order upwind schemes (UB-5, UB-7 and CU-5) typically reached a statistically stationary state within five flowthrough times, while the UF-2 and UB-3 schemes, particularly on the coarser grids, typically took longer. The flow laminarized in some of the upwind cases: UB- 1 on all grids, UB- 3 on the $48 \times 129 \times 48$ point grid, and UF- 2 on the $64 \times 129 \times 64$ and $48 \times 129 \times 48$ point grids. All other upwind cases achieved a statistically stationary turbulent state, and the same averaging process as had been applied in the central difference cases was performed over 15 flowthrough times.

Because relatively little change in the averaged flowfield profiles occurred when both halves of the channel were combined together into one profile, all subsequent results shown will depict the combined average. Timeand space-averaged flowfield values are denoted by an overbar (i.e. $\bar{u}, \bar{\rho}_{w}, \bar{\mu}_{w}$ and $\bar{u}_{\tau}=\sqrt{\bar{\tau}_{x y, w} / \bar{\rho}_{w}}$, where $\left.\bar{\tau}_{x y, w}=\bar{\mu}_{w}|\partial \bar{u} / \partial y|_{w}\right)$. Time- and space-averaged flowfield variances and covariances are denoted by a prime and an overbar (i.e. $\bar{u}^{\prime 2}, \bar{v}^{\prime 2}, \bar{w}^{\prime 2}$ and $\overline{u^{\prime} v^{\prime}}$ ).

The wall variable normalized velocity profiles for all schemes on the $128 \times 129 \times 128$ point grid are shown in the top panel of figure 8 . The velocity profiles are normalized by the characteristic wall friction velocity, $\bar{u}^{+}=$ $\bar{u} / \bar{u}_{\tau}$, and the distance from the wall is normalized by the wall friction lengthscale, $y^{+}=\left|y-y_{w}\right| \bar{u}_{\tau} \bar{\rho}_{w} / \bar{\mu}_{w}$. Profiles are also shown for the laminar sublayer, $u^{+}=y^{+}$and the log layer, $u^{+}=2.44 \ln \left(y^{+}\right)+5.2$. Also shown is the velocity profile from the $R e_{\tau}=395$ DNS calculation in Ref. 34. In the middle panel of figure 8, profiles of the normalized square root of the variance (RMS) for each velocity component, $\bar{u}^{\prime+}=\sqrt{\bar{u}^{\prime 2}} / \bar{u}_{\tau}$, $\bar{v}^{\prime+}=\sqrt{\bar{v}^{\prime 2}} / \bar{u}_{\tau}$, and $\bar{w}^{\prime+}=\sqrt{\bar{w}^{\prime 2}} / \bar{u}_{\tau}$ are shown. Profiles of the normalized turbulent shear stress, $-\overline{u^{\prime} v^{\prime}} / \bar{u}_{\tau}^{2}$ are shown in the lower panel of the figure. Observe that all five of the central difference schemes (left column of the figure) are in generally excellent agreement with the DNS calculation for all of the averaged flowfield profiles at this grid resolution. All of them slightly underpredict the peak of $\bar{u}^{\prime+}$, but in general they follow the DNS profile quite closely. For reference, the grid spacings normalized by the wall friction lengthscale for the central difference schemes are: $\Delta x^{+}=\Delta x \bar{u}_{\tau} \bar{\rho}_{w} / \bar{\mu}_{w}=20, \Delta y_{w}^{+}=0.42, \Delta y_{c}^{+}=16$ and $\Delta z^{+}=10$. 

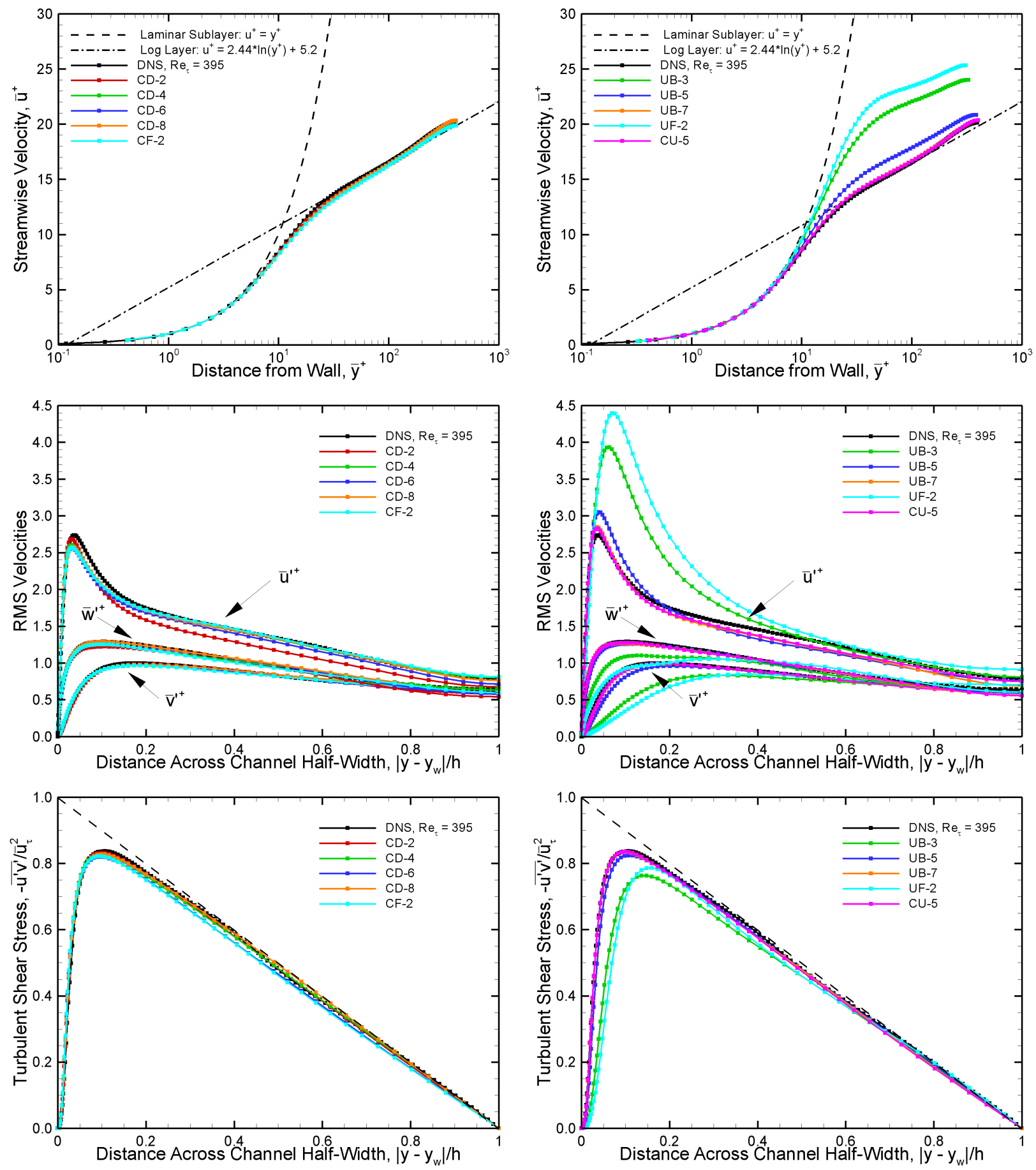

Figure 8. Comparison of time- and space-averaged profiles for the inviscid flux schemes on a $128 \times 129 \times 128$ point grid. Left column: central schemes. Right column: upwind schemes

Turning to the upwind schemes (right column of figure 8), both UB-7 and CU-5 also generally agree very well with the DNS profiles at this grid resolution. In contrast, UB-5, UB-3 and UF-2 have progressively worse agreement with the DNS profiles. The $\bar{u}^{+}$profiles rise increasingly above the log law, while $\bar{u}^{\prime+}$ progressively peaks at a higher value further away from the wall. The peaks of $\bar{v}^{\prime+}$ and $\bar{w}^{\prime+}$ progressively decrease. The displacement of the profile for $-\overline{u^{\prime} v^{\prime}} / \bar{u}_{\tau}^{2}$ for UF-2 and UB-3 is also evident. As UB-1 laminarized the flow, it is not shown in this figure.

The same comparison in averaged profiles is shown for all schemes on the $48 \times 129 \times 48$ point grid in 

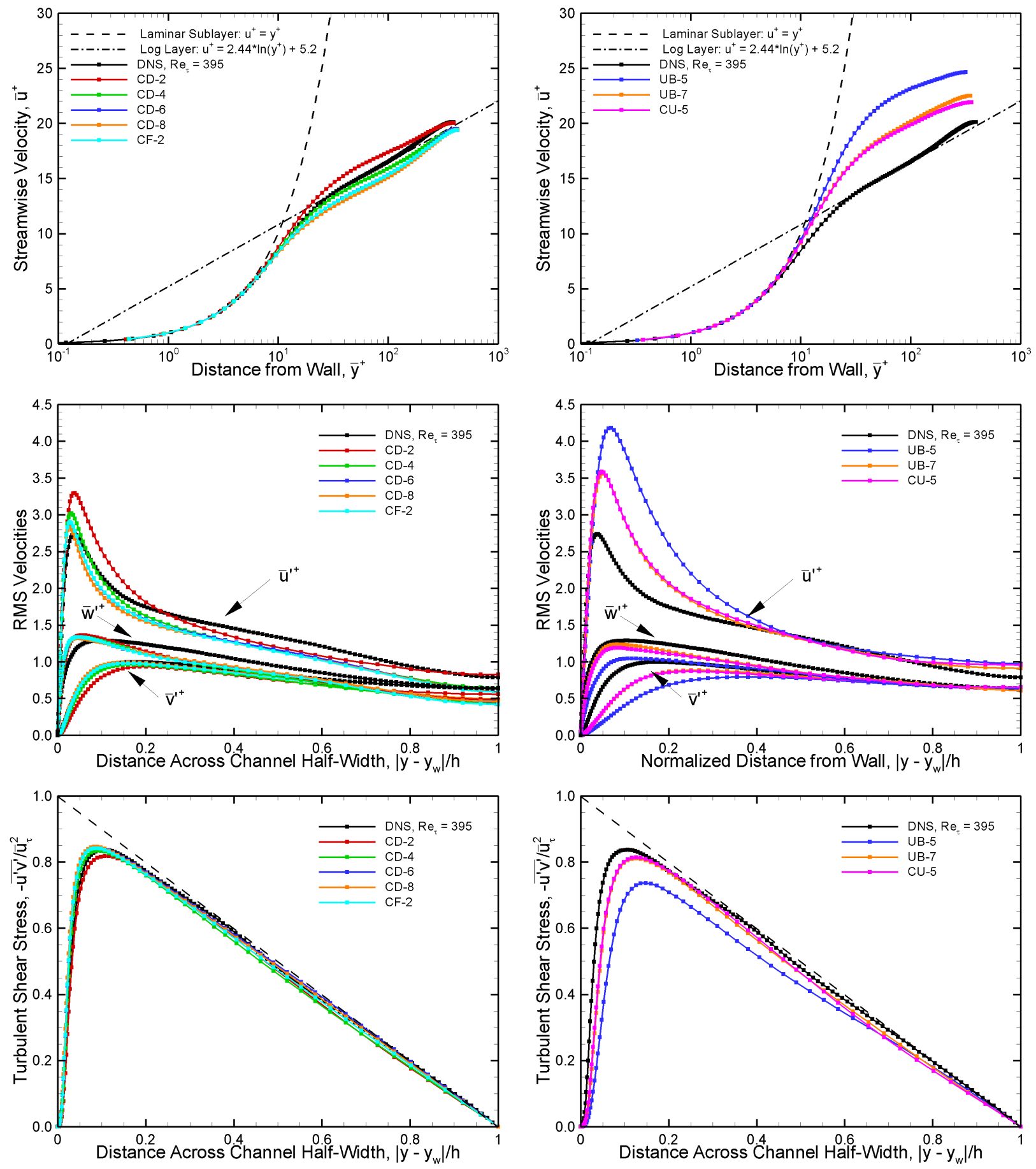

Figure 9. Comparison of time- and space-averaged profiles for the inviscid flux schemes on a $48 \times 129 \times 48$ point grid. Left column: central schemes. Right column: upwind schemes

figure 9. All schemes generally do poorer at this coarse grid resolution. CD-6, CD-8 and CF-2 have a $\bar{u}^{+}$ profile which lies consistently below the log law and DNS profile, while CD-2 and CD-4 are overall closer to the log law but have an erroneous slope. As an LES SGS model will typically adjust the velocity profile upward, use of such a model with the CD-6, CD-8 and CF-2 schemes would likely improve agreement with the DNS, while the CD-2 and CD-4 schemes would not likely benefit. The central scheme profiles of $\bar{u}^{\prime+}$, $\bar{v}^{\prime+}$ and $\bar{w}^{\prime+}$ diverge considerably from the reference DNS, and particularly so for CD-2.

Only profiles for UB-5, UB-7 and CU-5 are shown in the right column of figure 9 , as all of the other 

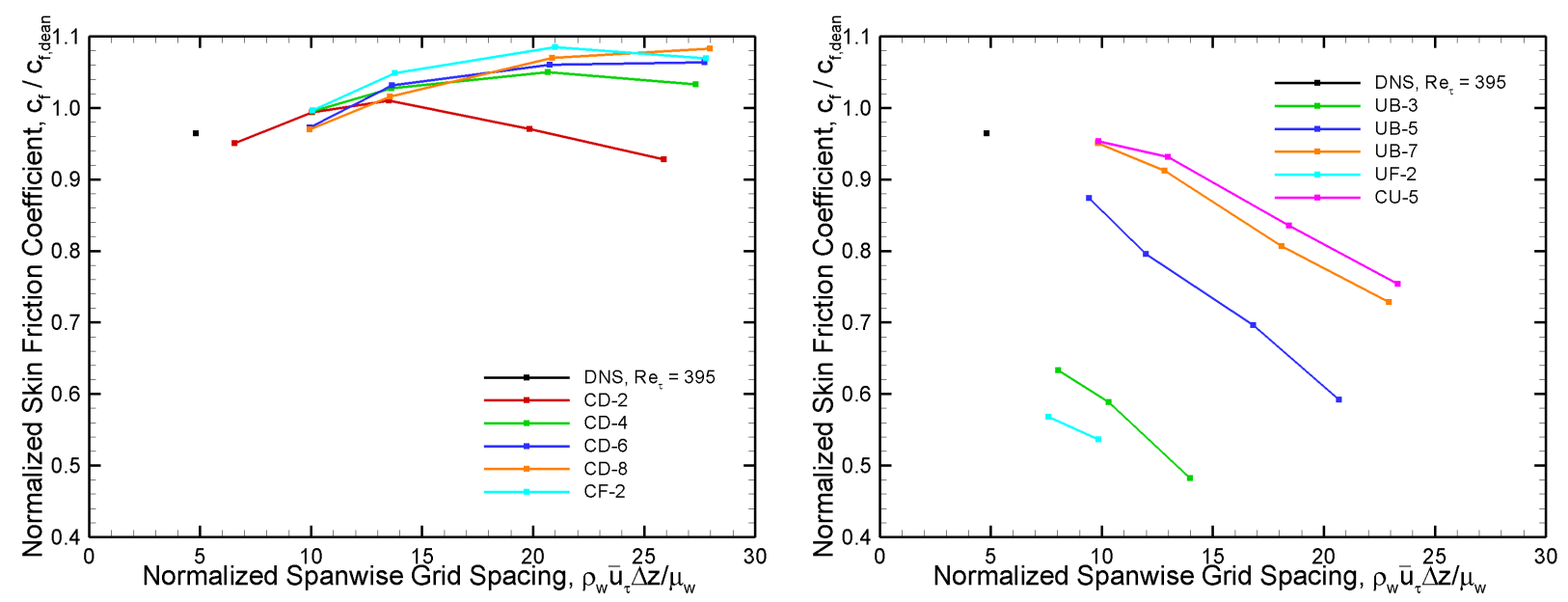

Figure 10. Effect of normalized spanwise grid spacing, $\Delta z^{+}=\Delta z \bar{u}_{\tau} \bar{\rho}_{w} / \bar{\mu}_{w}$ on the ratio of the skin friction coefficient, $c_{f}$, to the corresponding $c_{f}$ predicted by the Dean relation. Left panel: central schemes. Right panel: upwind schemes

upwind schemes laminarized the flow. UB-5 at this grid resolution yields results similar to those for UB-3 and UF-2 on the $128 \times 129 \times 128$ point grid. UB-7 and CU-5 are significantly better, but still not very close to the reference DNS profiles.

Another way to visualize the characteristics of these flux schemes as the grid resolution is decreased is shown in figure 10 . The ratio of the average skin friction coefficient, $c_{f}=\bar{\tau}_{x y, w} / \frac{1}{2} \bar{\rho}_{m} \bar{u}_{m}^{2}$, to the corresponding value of the skin friction coefficient calculated by the Dean expression, ${ }^{32} c_{f \text {,dean }}$, is plotted as a function of wall-normalized grid spacing in the spanwise $z$-direction, $\Delta z^{+}=\Delta z \bar{u}_{\tau} \bar{\rho}_{w} / \bar{\mu}_{w}$. The Dean expression for skin friction was developed from a literature survey of experimental data, and is

$$
c_{f, \text { dean }}=0.073 R e_{2 h}^{-0.25}
$$

where $R e_{2 h}$ is calculated from $\bar{\rho}_{m}, \bar{u}_{m}$ and $\bar{\mu}_{m}$ for each case, but is typically 14260 . $c_{f \text {,dean for these cases }}$ is $6.68 \times 10^{-3}$. Also shown in the figure is the result for the reference DNS calculation. Note that the CD-2 scheme has an additional data point compared to the others, which results from the single simulation carried out on the $192 \times 193 \times 192$ point grid. Simulations that resulted in laminarized flow are not plotted.

It is evident from figure 10 that the central schemes provide a good prediction of the skin friction for $\Delta z^{+} \leq 10$. At larger values of $\Delta z^{+}$, the central difference schemes begin to overpredict the skin friction. This is due to the lack of grid resolution for resolving the small turbulent lengthscales near the wall, and typically an LES SGS model would be employed in these cases to model the contribution of the small scales to the flow. While for most of the CD schemes this upward trend continues on the progressively coarser grids, note that the CD-2 scheme begins to underpredict the skin friction on the coarsest grids. The relatively poor dispersion accuracy of CD-2 on comparison to the other central difference schemes is likely the reason for this.

Considering the upwind schemes, consistent with the results shown previously, UB-7 and CU-5 also appear to provide a good prediction of skin friction for $\Delta z^{+} \leq 10$, and even provide a reasonable prediction (within 10\%) for $\Delta z^{+} \leq 13$. The predictive accuracy of even these two upwind schemes rapidly falls off at larger grid spacings however. Extrapolating the line for UB-5, it is reasonable to expect good predictive accuracy for this scheme for $\Delta z^{+} \approx 5$. However, it is not within $10 \%$ for any of the grid resolutions studied in this work. UB-3 and UF-2 are very poor in predictive accuracy.

\section{Conclusions}

Five different central difference schemes, based on a conservative differencing form of the Kennedy and Gruber skew-symmetric scheme, were tested and compared with six different upwind schemes based on primitive variable reconstruction and the Roe flux. These eleven different inviscid flux schemes were tested on a simple one-dimension acoustic standing wave problem, the Taylor-Green vortex problem, and a turbulent channel flow problem. 
In general, all of the central difference inviscid flux schemes studied proved to be generally very accurate and stable, provided that the grid stretching rate was kept below approximately 10\%. At near-DNS grid resolutions, these schemes yielded numerical results comparable to reference DNS results computed with spectral codes. At coarser resolutions, the need for an LES SGS model became apparent for these schemes. Consistent with a fourier analysis of the dispersion characteristics of these schemes, there was a noticeable increase in the accuracy of the results moving from second to fourth order accuracy. There appear to be clear benefits to moving to moving to higher dispersion accuracy, particularly at coarser grid resolutions.

The seventh order upwind biased scheme, and the fifth order compact upwind scheme, also performed very well at near-DNS grid resolutions. The fifth order upwind biased scheme does not do as well, but does appear to be suitable for well-resolved DNS. Second and third order upwind schemes having large dissipation ranges appear to be poorly suited for DNS or LES.

\section{References}

${ }^{1}$ Moin, P., "Numerical and Physical Issues in Large Eddy Simulation of Turbulent Flows," JSME International Journal, Series B, Vol. 41, No. 2, 1998, pp. $454-463$.

${ }^{2}$ Fureby, C., "Towards the use of large eddy simulation in engineering," Progress in Aerospace Sciences, Vol. 44, No. 6, 2008 , pp. $381-396$.

${ }^{3}$ Georgiadis, N. J., Rizzetta, D. P., and Fureby, C., "Large-Eddy Simulation: Current Capabilities, Recommended Practices, and Future Research," AIAA Journal, Vol. 48, No. 8, 2010, pp. 1772 - 1784.

${ }^{4}$ Spalart, P. R., Jou, W.-H., Strelets, M., and Allmaras, S. R., "Comments on the feasibility of LES for wings, and on a hybrid RANS/LES approach," In "Advances in DNS/LES: proceedings of the First AFOSR International Conference on DNS/LES", C. Liu and Z. Liu Eds., Greyden Press, Columbus, OH.

${ }^{5}$ Mittal, R. and Moin, P., "Suitability of Upwind-Biased Finite Difference Schemes for Large-Eddy Simulation of Turbulent Flows," AIAA Journal, Vol. 35, No. 8, 1997, pp. 1415 - 1417.

${ }^{6}$ Garnier, E., Mossi, M., Sagaut, P., Comte, P., and Deville, M., "On the Use of Shock-Capturing Schemes for Large-Eddy Simulation," Journal of Computational Physics, Vol. 153, No. 2, 1999, pp. 273 - 311.

${ }^{7}$ Mossi, M. and Sagaut, P., "Numerical investigation of fully developed channel flow using shock-capturing schemes," Computers 85 Fluids, Vol. 32, No. 2, 2003, pp. 249 - 274.

${ }^{8}$ Park, N., Yoo, J. Y., and Choi, H., "Discretization errors in large eddy simulation: on the suitability of centered and upwind-biased compact difference schemes," Journal of Computational Physics, Vol. 198, No. 2, 2004, pp. 580 - 616.

${ }^{9}$ Ducros, F., Laporte, F., Soulres, T., Guinot, V., Moinat, P., and Caruelle, B., "High-Order Fluxes for Conservative Skew-Symmetric-like Schemes in Structured Meshes: Application to Compressible Flows," Journal of Computational Physics, Vol. 161, No. 1, 2000, pp. $114-139$.

${ }^{10}$ Ducros, F., Ferrand, V., Nicoud, F., Weber, C., Darracq, D., Gacherieu, C., and Poinsot, T., "Large-Eddy Simulation of the Shock/Turbulence Interaction," Journal of Computational Physics, Vol. 152, No. 2, 1999, pp. $517-549$.

${ }^{11}$ Honein, A. E. and Moin, P., "Higher entropy conservation and numerical stability of compressible turbulence simulations," Journal of Computational Physics, Vol. 201, No. 2, 2004, pp. $531-545$.

${ }^{12}$ Larsson, J., Lele, S. K., and Moin, P., "Effect of numerical dissipation on the predicted spectra for compressible turbulence," Technical Report, Annual Research Briefs, Center for Turbulence Research, Stanford University, 2007.

${ }^{13}$ Johnsen, E., Larsson, J., Bhagatwala, A. V., Cabot, W. H., Moin, P., Olson, B. J., Rawat, P. S., Shankar, S. K., Sjögreen, B., Yee, H., Zhong, X., and Lele, S. K., "Assessment of high-resolution methods for numerical simulations of compressible turbulence with shock waves," Journal of Computational Physics, Vol. 229, No. 4, 2010, pp. 1213 - 1237.

${ }^{14}$ Pirozzoli, S., "Numerical Methods for High-Speed Flows," Annual Review of Fluid Mechanics, Vol. 43, No. 1, 2011, pp. $163-194$.

${ }^{15}$ Morris, C. I. and Ruf, J. H., "Validation of Supersonic Film Cooling Modeling for Liquid Rocket Engine Applications," AIAA Paper 2010-6657, July 2010.

${ }^{16}$ White, F. M., Viscous Fluid Flow, McGraw-Hill, New York, 2nd ed., 1991, pp. 26 - 32.

${ }^{17}$ Pirozzoli, S., "Conservative Hybrid Compact-WENO Schemes for Shock-Turbulence Interaction," Journal of Computational Physics, Vol. 178, No. 1, 2002, pp. $81-117$.

${ }^{18}$ Kennedy, C. A. and Gruber, A., "Reduced aliasing formulations of the convective terms within the NavierStokes equations for a compressible fluid," Journal of Computational Physics, Vol. 227, No. 3, 2008, pp. 1676 - 1700.

${ }^{19}$ Subbareddy, P. K. and Candler, G. V., "A fully discrete, kinetic energy consistent finite-volume scheme for compressible flows," Journal of Computational Physics, Vol. 228, No. 5, 2009, pp. $1347-1364$.

${ }^{20}$ Pirozzoli, S., "Generalized conservative approximations of split convective derivative operators," Journal of Computational Physics, Vol. 229, No. 19, 2010, pp. $7180-7190$.

${ }^{21}$ Pirozzoli, S., "Stabilized non-dissipative approximations of Euler equations in generalized curvilinear coordinates," Journal of Computational Physics, Vol. 230, No. 8, 2011, pp. 2997 - 3014.

${ }^{22}$ Adams, N. and Shariff, K., "A High-Resolution Hybrid Compact-ENO Scheme for Shock-Turbulence Interaction Problems," Journal of Computational Physics, Vol. 127, No. 1, 1996, pp. 27 - 51.

${ }^{23}$ Zhong, X., "High-Order Finite-Difference Schemes for Numerical Simulation of Hypersonic Boundary-Layer Transition," Journal of Computational Physics, Vol. 144, No. 2, 1998, pp. $662-709$.

${ }^{24}$ Gerolymos, G. A., Senechal, D., and Vallet, I., "DNS of Compressible Channel Flow using Low-Diffusion High-Order Upwind Schemes," AIAA Paper 2007-4196, June 2007. 
${ }^{25}$ Rehman, S. F., Eldredge, J. D., Zhong, X., and Kim, J., "A Simple and Robust Approach for Higher Order Hybrid Shock Capturing Methods," AIAA Paper 2010-4453, June 2010.

${ }^{26}$ Roe, P., "Approximate Riemann solvers, parameter vectors, and difference schemes," Journal of Computational Physics, Vol. 43, No. 2, 1981, pp. $357-372$.

${ }^{27}$ Vichnevetsky, R. and Bowles, J. B., Fourier Analysis of Numerical Approximations of Hyperbolic Equations, Society for Industrial and Applied Mathematics, Philadelphia, 1982, pp. 19 - 49.

${ }^{28} \mathrm{Li}$, Y., "Wavenumber-Extended High-Order Upwind-Biased Finite-Difference Schemes for Convective Scalar Transport," Journal of Computational Physics, Vol. 133, No. 2, 1997, pp. 235 - 255.

${ }^{29}$ Brachet, M. E., Meiron, D. I., Orszag, S. A., Nickel, B. G., Morf, R. H., and Frisch, U., "Small-scale structure of the Taylor-Green vortex," Journal of Fluid Mechanics, Vol. 130, 1983, pp. 411 - 452.

${ }^{30}$ van Rees, W. M., Leonard, A., Pullin, D., and Koumoutsakos, P., "A comparison of vortex and pseudo-spectral methods for the simulation of periodic vortical flows at high Reynolds numbers," Journal of Computational Physics, Vol. 230, No. 8, 2011 , pp. $2794-2805$.

${ }^{31}$ Hunt, J. C. R., Wray, A. A., and Moin, P., "Eddies, Streams, and Convergence Zones in Turbulent Flows," Proceedings of the 1988 Summer Program, Center for Turbulence Research, Stanford University, 1988, pp. $193-208$.

${ }^{32}$ Dean, R. B., "Reynolds Number Dependence of Skin Friction and Other Bulk Flow Variables in Two-Dimensional Rectangular Duct Flow," Journal of Fluids Engineering, Vol. 100, No. 2, 1978, pp. 215 - 223.

${ }^{33} \mathrm{Kim}$, J., Moin, P., and Moser, R., "Turbulence statistics in fully developed channel flow at low Reynolds number," Journal of Fluid Mechanics, Vol. 177, 1987, pp. 133 - 166.

${ }^{34}$ Moser, R. D., Kim, J., and Mansour, N. N., "Direct numerical simulation of turbulent channel flow up to $\operatorname{Re}_{\tau}=590$," Physics of Fluids, Vol. 11, No. 4, 1999, pp. 943 - 945.

${ }^{35}$ Choi, H. and Moin, P., "Effects of the Computational Time Step on Numerical Solutions of Turbulent Flow," Journal of Computational Physics, Vol. 113, No. 1, 1994, pp. $1-4$.

${ }^{36}$ Moin, P. and Kim, J., "On the numerical solution of time-dependent viscous incompressible fluid flows involving solid boundaries," Journal of Computational Physics, Vol. 35, No. 3, 1980, pp. 381 - 392. 